\title{
DEPINNING TRANSITIONS IN DISCRETE REACTION-DIFFUSION EQUATIONS*
}

\author{
A. CARPIO $^{\dagger}$ AND L. L. BONILLA $\ddagger$
}

\begin{abstract}
We consider spatially discrete bistable reaction-diffusion equations that admit wave front solutions. Depending on the parameters involved, such wave fronts appear to be pinned or to glide at a certain speed. We study the transition of traveling waves to steady solutions near threshold and give conditions for front pinning (propagation failure). The critical parameter values are characterized at the depinning transition, and an approximation for the front speed just beyond threshold is given.
\end{abstract}

Key words. discrete reaction-diffusion equations, traveling wave fronts, propagation failure, wave front depinning

AMS subject classifications. 34E15, 92C 30

PII. S003613990239006X

1. Introduction. Spatially discrete systems describe physical reality in many different fields: atoms adsorbed on a periodic substrate [13], motion of dislocations in crystals [32], propagation of cracks in a brittle material [35], microscopic theories of friction between solid bodies [18], propagation of nerve impulses along myelinated fibers [23, 24], pulse propagation through cardiac cells [24], calcium release waves in living cells [6], sliding of charge density waves [19], superconductor Josephson array junctions [39], or weakly coupled semiconductor superlattices $[3,9]$. No one really knows why, but spatially discrete systems of equations often have smooth solutions of the form $u_{n}(t)=u(n-c t)$, which are monotone functions approaching two different constants as $(n-c t) \rightarrow \pm \infty$. Existence of such wave front solutions has been proved for particular discrete systems having dissipative dynamics [40]. In the case of discrete systems with conservative dynamics, a wave front solution was explicitly constructed by Flach, Zolotaryuk, and Kladko [16]. However, a general proof of wave front existence for discrete conservative systems with bistable sources is lacking.

A distinctive feature of spatially discrete reaction-diffusion systems (not shared by continuous ones) is the phenomenon of wave front pinning: for values of a control parameter in a certain interval, wave fronts joining two different constant states fail to propagate [24]. When the control parameter surpasses a threshold, the wave front depins and starts moving $[23,19,32,9]$. The existence of such thresholds is thought to be an intrinsically discrete fact, which is lost in continuum approximations. The characterization of propagation failure and front depinning in discrete systems is thus an important problem, which is not yet well understood despite the numerous inroads made in the literature $[23,6,19,32,25,26,28,30,27,36,37,38]$.

*Received by the editors April 3, 2002; accepted for publication (in revised form) August 20, 2002; published electronically February 25, 2003. This research was supported by the Spanish MCyT grant BFM2002-04127-C02, by the Third Regional Research Program of the Autonomous Region of Madrid (Strategic Groups Action), and by the European Union under grant RTN2-2001-00349.

http://www.siam.org/journals/siap/63-3/39006.html

${ }^{\dagger}$ Departamento de Matemática Aplicada, Universidad Complutense de Madrid, 28040 Madrid, Spain (carpio@mat.ucm.es).

${ }^{\ddagger}$ Departamento de Matemáticas, Escuela Politécnica Superior, Universidad Carlos III de Madrid, Avenida de la Universidad 30, 28911 Leganés, Spain, and Unidad Asociada al Instituto de Ciencia de Materiales de Madrid (CSIC), 28049 Cantoblanco, Spain (bonilla@ing.uc3m.es). 
In this paper, we study front depinning for infinite one-dimensional nonlinear spatially discrete reaction-diffusion (RD) systems. When confronted with a spatially discrete RD system, a possible strategy is to approximate it by a continuous RD system. For generic nonlinearities, the width of the pinning interval is exponentially small as the continuum limit is approached. Pinning in the continuum limit has been analyzed by many authors using exponential asymptotics, also known as asymptotics beyond all orders. As far as we can tell, usage of these techniques for discrete equations goes back to two classic papers by Indenbom [22] (for the FK potential) and by Cahn [7] (for the double-well potential). In both cases, an exponential formula for the critical field was derived by means of the Poisson sum rule. In the context of dislocation motion, exponential formulas for the depinning shear stress of the PeierlsNabarro (PN) model were found earlier by Peierls [33] and Nabarro [31]. Descriptions of wave front pinning near the continuum limit can also be found in more recent work $[20,25,27]$.

Analyzing the continuum limit of a discrete system by means of exponential asymptotics is a costly strategy for describing pinning for two reasons. It is not numerically accurate as we move away from the continuum limit, and it ceases to be useful if convective terms [11] or disorder [12] alter the structure of the discrete system (quite common in applications). Thus other authors have tried to describe the opposite strongly discrete limit. For discrete RD equations, Erneux and Nicolis [14] studied a finite discrete RD equation with a cubic nonlinearity, a Dirichlet boundary condition at one end, and a Neumann boundary condition at the other end. They considered a particular limit in which two of the three zeroes of the cubic nonlinearity coalesced as diffusivity went to zero. Erneux and Nicolis's calculation is essentially a particular case of our active point approximation that involves only one active point and makes an additional assumption on the nonlinearity (not needed in our calculations). They found that the wave front velocity scales as the square root of $\left(d-d_{c}\right)(d$ is the diffusivity and $d_{c}$ its critical value at which wave fronts are pinned). Essentially the same results can be found in the appendix of [27]. Kladko, Mitkov, and Bishop [28] introduced an approximation called the single active site theory. In this approximation, the wave front is described by two linear tails (solution of the RD equation linearized about each of the two constants joined by the front) patched at one point. This approximation is used to estimate the critical field for wave front depinning.

By a combination of numerical and asymptotic calculations, we arrive at the following description [10, 11]. The nature of the depinning transition depends on the nonlinearity of the model and is best understood as propagation failure of the traveling front. Usually, but not always, the wave front profiles become less smooth as a parameter $F$ (external field) decreases. They become discontinuous at a critical value $F_{c}$. Below $F_{c}$, the front is pinned at discrete positions corresponding to a stable steady state. As a consequence of the maximum principle for spatially discretized parabolic equations, stationary and moving wave fronts cannot simultaneously exist for the same value of $F$ (see [8]). This is not the case for chains with conservative dynamics, which are spatially discretized hyperbolic equations without a maximum principle. For chains with conservative Hamiltonian dynamics, an inverse method due to Flach, Zolotaryuk, and Kladko [16] explicitly shows that stationary and moving wave fronts may coexist for the same value of the parameters.

We consider chains of diffusively coupled overdamped oscillators in a potential $V$, subject to a constant external force $F$ :

$$
\frac{d u_{n}}{d t}=u_{n+1}-2 u_{n}+u_{n-1}+F-A g\left(u_{n}\right) .
$$



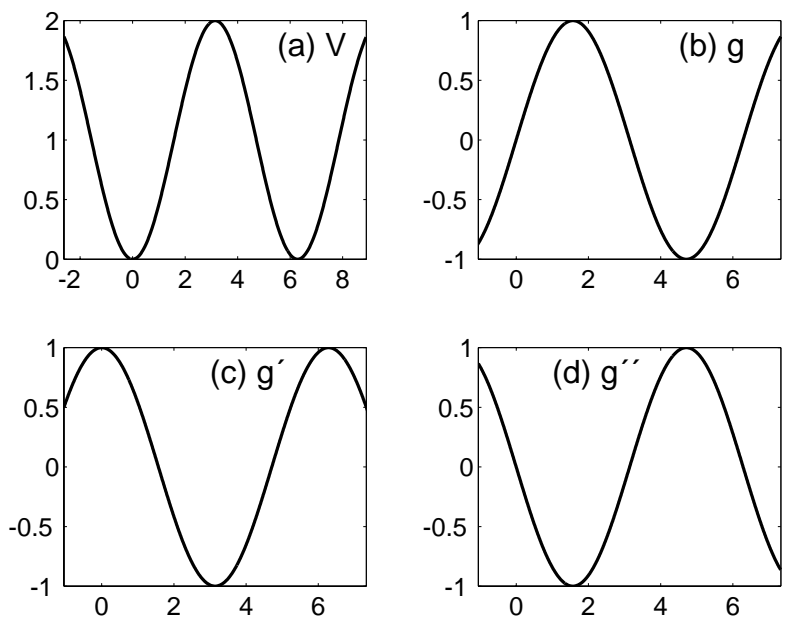

FIG. 1.1. FK model: (a) potential $1-\cos (x)$, (b) source term $g(u)=\sin (u)$, (c) $g^{\prime}(u)=\cos (u)$, (d) $g^{\prime \prime}(u)=-\sin (u)$.

Here $g(u)=V^{\prime}(u)$ is at least $C^{1}$, and it presents a "cubic" nonlinearity (see Figure 1.1), such that $A g(u)-F$ has three zeroes, $U_{1}(F / A)<U_{2}(F / A)<U_{3}(F / A)$ in a certain force interval $\left(g^{\prime}\left(U_{i}(F / A)\right)>0\right.$ for $\left.i=1,3, g^{\prime}\left(U_{2}(F / A)\right)<0\right)$. Provided that $g(u)$ is odd with respect to $U_{2}(0)$, there is a symmetric interval $|F| \leq F_{c}$ where the discrete wave fronts joining the stable zeroes $U_{1}(F / A)$ and $U_{3}(F / A)$ are pinned $[23,8]$. For $|F|>F_{c}$, there are smooth traveling wave fronts, $u_{n}(t)=u(n-c t)$, with $u(-\infty)=U_{1}$ and $u(\infty)=U_{3}$, as proved in $[40,8]$. The velocity $c(A, F)$ depends on $A$ and $F$, and it satisfies $c F<0$ and $c \rightarrow 0$ as $|F| \rightarrow F_{c}$ (see [8]). Examples are the overdamped Frenkel-Kontorova (FK) model $(g=\sin u$; see Figure 1.1) [17] and the quartic double well potential $\left(V=\left(u^{2}-1\right)^{2} / 4\right)$. Less symmetric nonlinearities yield a nonsymmetric pinning interval, and our analysis applies to them with trivial modifications. Note that coexistence of fronts traveling in opposite directions can occur in the case of conservative systems, but not for (1.1) due to the maximum principle (which is the basis of comparison techniques) [8].

For the overdamped FK model given by (1.1) with $g=\sin u$, Figure 1.2 shows wave front profiles near the critical field. Individual points undergo abrupt jumps at particular times, which gives the misleading impression that the motion of the discrete fronts proceeds by successive jumps. Actually, the points remain very close to their stationary values at $F=F_{c}$, say $u_{n}\left(A, F_{c}\right)$, during a very long time interval of order $\left|F-F_{c}\right|^{-\frac{1}{2}}$. Then, at a specific time, all the points $u_{n}(t)$ jump to a vicinity of $u_{n+1}\left(A, F_{c}\right)$. The method of matched asymptotic expansions can be used to describe this two-stage motion of the points $u_{n}(t)$. Then the wave front profile can be reconstructed by using the definition $u_{n}(t)=u(n-c t)$. The slow stage of front motion is described by the normal form of a saddle-node bifurcation, and it yields an approximation to the wave front velocity, which scales with the field as $\left|F-F_{c}\right|^{\frac{1}{2}}$. This scaling has been mentioned by other authors: it was found numerically in [1], and by means of exponential asymptotics in the limit $A$ small in [27]. It is also conjectured in [26] on the correct basis that the depinning transition consists of a saddle-node bifurcation (a similar claim was stated in [30] for continuous reaction diffusion equations with localized sources). However, the derivation of the local saddle-node normal form and 

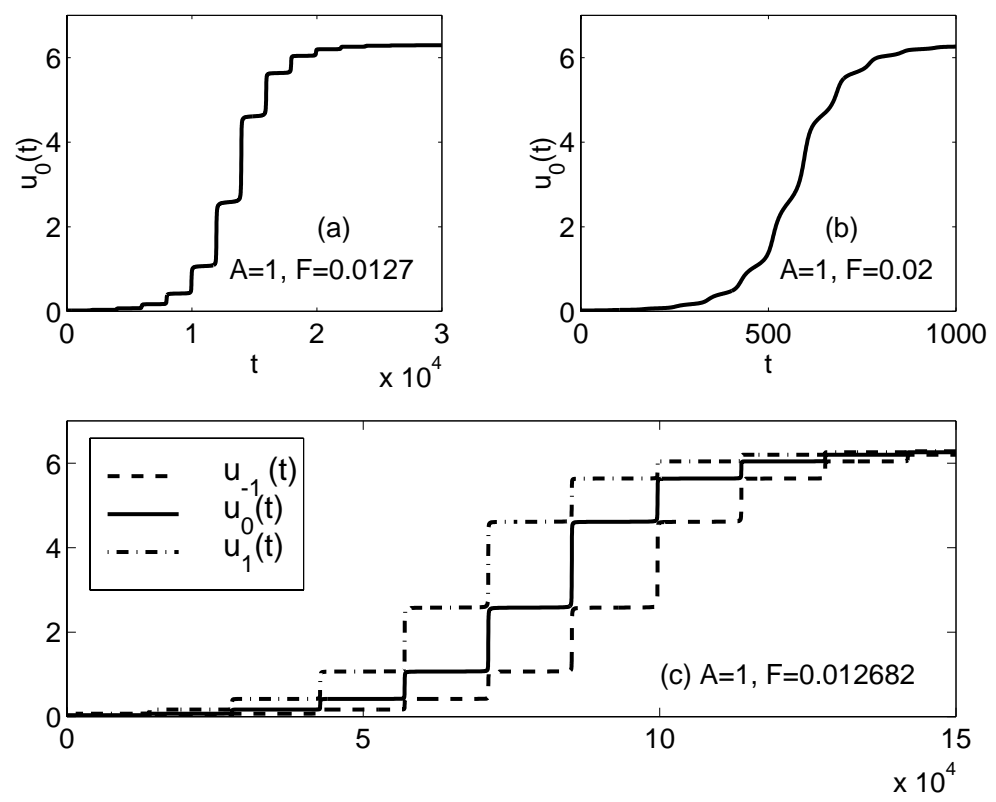

Fig. 1.2. Wave front profiles for the overdamped $F K$ model when $A=1$ near $F_{c}$.

the correct description of the global saddle-node bifurcation involving matching with a fast stage during which the front jumps abruptly one lattice period were apparently omitted by the authors of [26], who used energy arguments. Our picture of the wave front depinning transition has essentially been corroborated in the continuum limit (as an appropriate dimensionless lattice length goes to zero) by King and Chapman, who used asymptotics beyond all orders [27]. An independent confirmation follows from Fáth's calculations for a spatially discrete reaction-diffusion equation with a piecewise linear source term [15] (except that the velocity should scale differently with $\left|F-F_{c}\right|$ in this case).

For exceptional nonlinearities, the wave front does not lose continuity as the field decreases. In this case, there is a continuous transition between wave fronts moving to the left for $F>0$ and moving to the right for $F<0$; as for continuous systems, front pinning occurs at only a single field value $F=0$ (see $[27,16,36$, $37,38]$ ). Wave front velocity then scales linearly with the field. We discuss the characterization of the critical field (including analytical formulas in the strongly discrete limit), describe depinning anomalies (discrete systems having zero critical field $[36,37,38,16])$, and give a precise characterization of stationary and moving fronts near depinning (including front velocity) by singular perturbation methods. Our approximations show excellent agreement with numerical simulations.

The rest of the paper is organized as follows. In section 2, we characterize wave front depinning. We also explain that pinning of wave fronts normally occurs at force values belonging to an interval with nonzero length. However, there are nonlinearities for which pinning occurs only at $F=F_{c}=0$. In section 3 , we present a theory of wave front depinning for the strongly discrete case ( $A$ large). This theory enables us to predict the critical field and the speed and shape of the wave fronts near threshold. The main ideas of our theory are very simple. First, a wave front profile $u_{n}(t)=$ 
$u(n-c t)$ can be reconstructed if we follow the motion of one point during a sufficiently long time interval. Secondly, the analysis of (1.1) is complicated by the presence of the discrete diffusion term $u_{n+1}-2 u_{n}+u_{n-1}$. Previous authors have tried to approximate this term by its continuum limit (corresponding to $A \rightarrow 0$ ), which leads to using exponential asymptotics [27]. (See also [25] on using exponential asymptotics for the Hamiltonian version of our model.) However, we are only interested in constructing solutions of (1.1) joining constant values. For sufficiently large $A$ (say, $A=0.1$ for the FK model $), u_{i}$ is approximately either $U_{1}(F / A)$ or $U_{3}(F / A)$ except for a finite number of points (the active points). Then we can approximate the infinite system (1.1) by a closed system of ordinary differential equations (only one equation for $A \geq 10$ in the FK model). The depinning transition is a global bifurcation of this system, as explained in section 3. Some auxiliary technical results are collected in the appendix.

2. Front pinning as propagation failure. To describe monotone stationary solutions of (1.1) joining $U_{1}(F / A)$ and $U_{3}(F / A)$ for $|F| \leq F_{c}$, it is better to start by considering traveling wave fronts for $|F|>F_{c}$. It has been proved (and corroborated by numerical calculations) that traveling wave fronts and stationary profiles cannot coexist at the same value of $F$ (see [9]). Furthermore, numerical computations of wave fronts near the critical fields $F_{c}$ for the FK and other usual potentials show staircase-like wave front profiles, which sharpen as $F$ approaches $F_{c}$. At $F=F_{c}$, a series of gaps open up, and one is left with a discontinuous stationary profile $s(x)$ solving

$$
\begin{array}{r}
s(x+1)-2 s(x)+s(x-1)=A g(s(x))-F_{c}, \quad x \in \mathbb{R}, \\
s(-\infty)=U_{1}\left(\frac{F_{c}}{A}\right), \quad s(\infty)=U_{3}\left(\frac{F_{c}}{A}\right) .
\end{array}
$$

The profile $s(x)$ is increasing and piecewise constant. The sequence of constant values attained by $s(x)$ defines a steady solution $u_{n}$ of (1.1) with $F=F_{c}$. A stationary solution can thus be understood as a wave front that fails to propagate and is pinned at discrete values. Figure 1.2 illustrates the pinning transition for the FK model with $A=1$. As $F$ decreases from 0.02 to 0.0127 , a series of steps are formed. Figure 1.2(c) depicts the paths described by three consecutive points. All profiles look identical and are obtained by shifting any one of them some multiple of a certain constant length. This implies that the length of all steps in the profile is the same and that all the points $u_{n}(t)$ in (1.1) proceed to climb the next step in the staircase at the same time. This behavior indicates that the wave front is a traveling wave, $u_{n}(t)=u(n-c t)$. Proofs of this fact for some sources can be found in [40].

2.1. Limiting front profile at the critical field. Let us start by showing that the limit of the traveling waves as $F \rightarrow F_{c}$ is singular if $F_{c}>0$. This fact can be guessed from the differential-difference equations satisfied by the wave profiles. The traveling waves for $|F|>F_{c}$ have the form $u_{n}(t)=u(n-c t)$, where the profile $u(z)$ solves (see [8])

$$
\begin{array}{r}
-c u_{z}=u(z+1)-2 u(z)+u(z-1)-A g(u(z))+F, \quad z \in \mathbb{R}, \\
u(-\infty)=U_{1}\left(\frac{F}{A}\right), \quad u(\infty)=U_{3}\left(\frac{F}{A}\right) .
\end{array}
$$

The solution $u$ is as smooth as allowed by $g(u)$. ( $u$ is $C^{k+1}$ if $g(u)$ is $C^{k}$, with $k \geq 1$.) Then multiplying (2.2) by $u_{z}$ and integrating it, we get

$$
-c \int_{-\infty}^{\infty} u_{z}^{2} d z=F\left[U_{3}\left(\frac{F}{A}\right)-U_{1}\left(\frac{F}{A}\right)\right] .
$$


A first obvious conclusion is that the sign of $c$ is opposite to the sign of $F$. Let $F_{c}$ be positive. As $F \rightarrow F_{c}, c \rightarrow 0$ and $F\left[U_{3}(F / A)-U_{1}(F / A)\right] \rightarrow F_{c}\left[U_{3}\left(F_{c} / A\right)-\right.$ $\left.U_{1}\left(F_{c} / A\right)\right] \neq 0$. Therefore the integrals $\int u_{z}^{2} d z \rightarrow \infty$ as $F \rightarrow F_{c}$. Thus the limiting profile must be discontinuous if $F_{c}>0$.

If $F_{c}=0$, the relation (2.3) can be used to show that (2.1) has a smooth solution. In fact, provided that $c \sim-K F(K>0)$ as $F \rightarrow 0$, we can use (2.3) to uniformly bound the derivatives of the solutions $u$ in $(2.2)$ for $F \neq 0$. Then we obtain a smooth solution of (2.1) in the limit as $F \rightarrow 0$. We will come back to this question later on in subsection 2.3. Note that the stationary equation $s(x+1)-2 s(x)+s(x-1)=A g(s)-F$ has no continuous solutions joining $U_{1}(F / A)$ to $U_{3}(F / A)$ unless $F=0$. To see this [8], we multiply the equation by $s_{x}$ (in the sense of distributions if necessary) and integrate to get $F=0$.

2.2. Characterization of the critical field. Some results are available in the continuum limit $A \rightarrow 0$. For $g=\sin u$, it is well known that $F_{c}$ vanishes exponentially fast as $A$ goes to zero. An exponential formula for $F_{c}$ was first found by Indenbom [22] using the Poisson sum rule (following the calculations of the PN energy barrier for the PN model by Peierls [33] and Nabarro [31]) and numerically checked by Hobart [21] in the context of the Peierls stress and energy for dislocations. For the discrete bistable RD equation, Cahn [7] derived an exponential dependence of $F_{c}$ by a similar technique. Related ideas can be found in Kladko, Mitkov, and Bishop [28]. These arguments can be used for other potentials and suggest that $F_{c} \sim C e^{-\eta / \sqrt{A}}$ as $A \rightarrow 0+$ (with positive $C$ and $\eta$ independent of $A$ ) holds for a large class of nonlinearities. Using exponential asymptotics, King and Chapman [27] have obtained precise formulas for the critical field and the wave front velocity of a discrete RD equation. Particularized to the FK potential, their formulas for the critical field and for the wave front velocity after depinning are $F_{c} \sim \Lambda e^{-\pi^{2} /\left[2 \sinh ^{-1}(\sqrt{A} / 2)\right]}, \Lambda \approx 356.1$, and $c \sim D \sqrt{\left(F^{2}-F_{c}^{2}\right) / A}$, respectively. This latter result agrees with the scaling law $c \sim\left|F-F_{c}\right|^{\frac{1}{2}}$, found in a large class of discrete RD equations $[9,10,11,26]$ and in continuous equations with localized sources [30]. However, exponential asymptotics [27] does not work for $A$ large. We shall therefore follow a different approach. We shall begin by considering stationary increasing discrete front profiles and study under which conditions they start moving. Since stationary fronts are pinned wave fronts, we can call the transition from stationary to moving fronts the depinning transition.

Two facts distinguish the depinning transition: (i) the smallest eigenvalue of (1.1) linearized about a stable stationary profile becomes zero (see below), and (ii) stationary and moving wave fronts cannot coexist for the same values of the field. First, the following comparison principle [23] for (1.1) can be used to show that stationary and traveling wave fronts cannot coexist for the same value of $F$ (see [8]).

COMPARISON PRINCIPLE. Assume that we have two configurations $w_{n}(t)$ and $l_{n}(t)$. If initially $w_{n}(0) \geq l_{n}(0)$ for all $n$, and at any later time $t>0$

$$
\begin{array}{r}
\frac{d w_{n}}{d t} \geq w_{n+1}-2 w_{n}+w_{n-1}-A g\left(w_{n}\right)+F, \\
\frac{d l_{n}}{d t} \leq l_{n+1}-2 l_{n}+l_{n-1}-A g\left(l_{n}\right)+F
\end{array}
$$

for all $n \in \mathbb{Z}$, then necessarily $w_{n}(t) \geq l_{n}(t)$ for all $n$ and $t$. Here $w_{n}$ satisfying (2.4) is said to be a supersolution, and $l_{n}$ satisfying (2.5) is said to be a subsolution.

Front pinning can be proved using stationary sub- and supersolutions, which can be constructed, provided that the stationary solution is linearly stable. The 

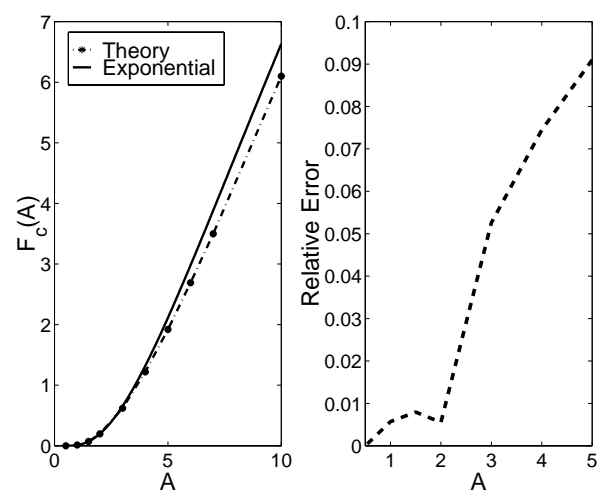

FIG. 2.1. (a) Critical field, $F_{c}(A)$, for $A \in\left(\frac{1}{2}, 10\right)$. We have compared the result of approximately solving $\lambda_{1}(A, F)=0$ for $F$ as a function of $A$ (see the appendix) to the asymptotic result $F_{c} \sim 356.1 e^{-\pi^{2} /\left[2 \sinh ^{-1}(\sqrt{A} / 2)\right]}$ of [27]. (b) Relative error of the exponential asymptotics approximation.

smallest eigenvalue of the linearization of (1.1) about a stationary profile $u_{n}(A, F)$, $u_{n}(t)=u_{n}(A, F)+v_{n} e^{-\lambda t}$, is given by

$$
\lambda_{1}(A, F)=\min \frac{\sum\left[\left(v_{n+1}-v_{n}\right)^{2}+A g^{\prime}\left(u_{n}(A, F)\right) v_{n}^{2}\right]}{\sum v_{n}^{2}},
$$

over a set of functions $v_{n}$, which decay exponentially as $n \rightarrow \pm \infty$. We show in the appendix that the minimum is attained at a positive eigenfunction.

The critical field can be uniquely characterized by $\lambda_{1}\left(A, F_{c}\right)=0$ and $\lambda_{1}(A, F)>0$ for $|F|<F_{c}$. The details are given in the appendix. Notice that $\lambda_{1}(A, F)>0$ implies that (2.1) does not have smooth solutions $s(x)$; otherwise, $v_{n}=s^{\prime}(n)$ is an eigenfunction corresponding to $\lambda_{1}=0$ as it happens in the continuum limit. The previous characterization is the basis of a procedure for calculating $F_{c}(A)$. In section 3 , we shall show that wave fronts near the depinning transition are described by a reduced system of equations for a finite number of points $u_{n}(t)$ which "jump" from about a discrete value corresponding to the stationary solution, $u_{n}\left(A, F_{c}\right)$, to the next one, $u_{n+1}\left(A, F_{c}\right)$, during front motion. The smallest eigenvalue for the linearization of the reduced system of equations about a stationary solution approximates $\lambda_{1}$ well. The critical field obtained by this procedure has been depicted in Figure 2.1 for the FK potential and compared to King and Chapman's asymptotic result (obtained by keeping two terms in their formulas). Notice that the asymptotic result loses accuracy as $A$ increases.

Equation (2.6) shows that the critical field is positive for large $A$ and typical nonlinearities. In fact, consider the FK potential. For $F=0$ there are two oneparameter families of stationary solutions which are symmetric with respect to $U_{2}$ (see Figure 2.2), one taking on the value $U_{2}$ (unstable dislocation), and the other one having $u_{n} \neq U_{2}$ (stable dislocation) $[21,8]$. The centers of two stable (or two unstable) dislocations differ in an integer number of lattice periods. Except for a possible rigid shift, the stable dislocation, $u_{n}(A, 0)$, is a dynamically stable stationary solution towards which step-like initial conditions evolve. Figures 2.3(a) and (b) show two initial conditions that evolve (exponentially fast) towards the stable dislocation. Half the initial points $u_{n}(0)$ have been selected to be below $U_{2}$, and the other half are above this value. In Figure 2.3(a), $u_{n}(0)-u_{n}(A, 0)=\epsilon_{n}$, where $\epsilon_{n}$ are real random 

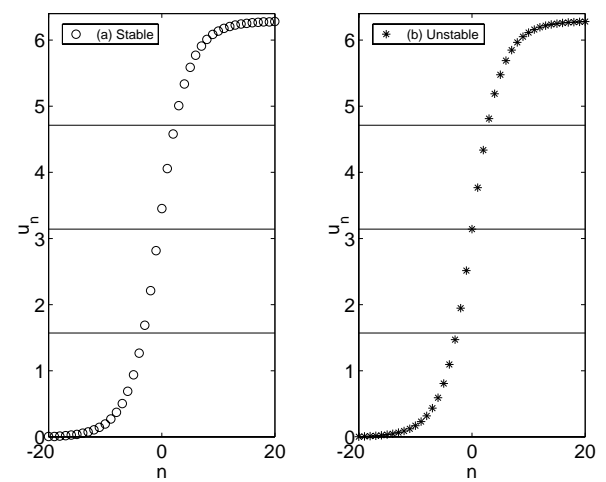

FIG. 2.2. Stable and unstable dislocations for the $F K$ model when $F=0$ and $A=0.1$.
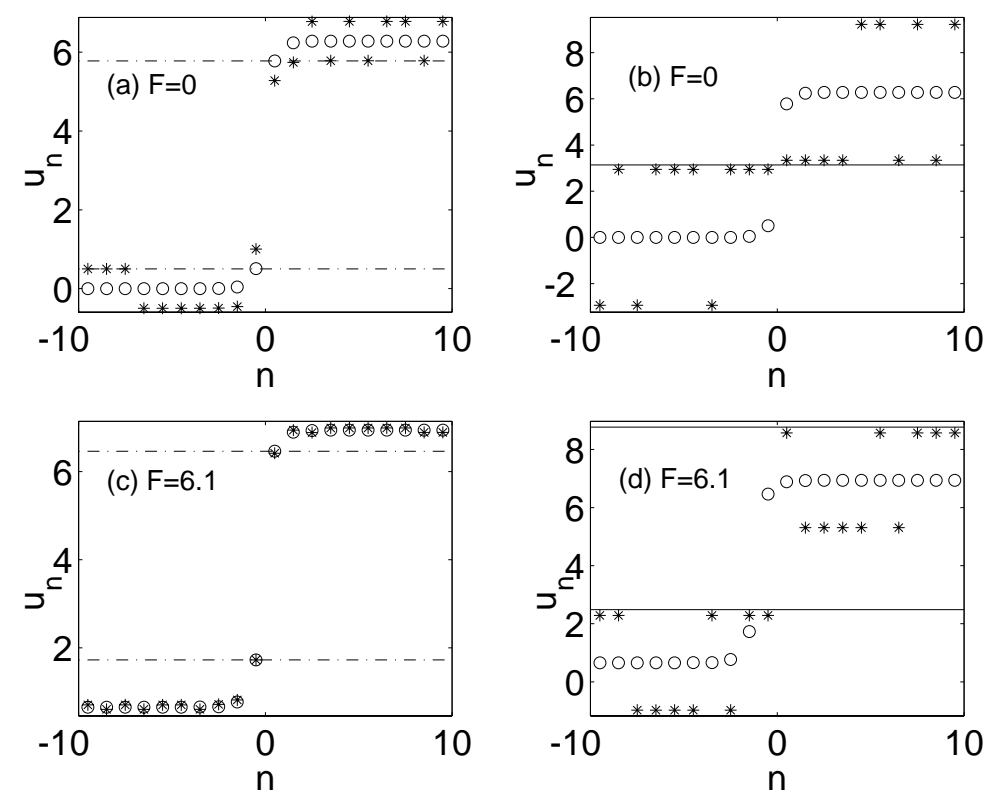

FIG. 2.3. Initial condition $u_{n}(0)$ (asterisks) and its large time limit, the stable dislocation (circles), for the FK model with $A=10 ; F=0$ for (a) and (b), and $F=6.1<F_{c}$ for (c) and (d). The initial points are selected as indicated in the text.

numbers with $\left|\epsilon_{n}\right|<0.5$. In Figure 2.3(b), $u_{n}(0)-U_{1,3}=\delta_{n} B, 0<B=U_{2}-U_{1}-0.2$, and $\delta_{n}$ randomly takes on the values 1 or -1 . By using comparison methods, it is possible to prove that a small disturbance of the stable dislocation evolves towards it. The same results hold for the stable stationary solution $u_{n}(A, F)$ for $0<|F|<F_{c}$. As $|F|$ increases, a disturbance of the stable stationary solution typically evolves towards the same stationary solution displaced an integer number of lattice periods unless the disturbance is sufficiently small. See Figure 2.3(d) for an example of this phenomenon for $F$ slightly smaller than $F_{c}$. Carefully selecting the initial condition avoids this, as in Figure 2.3(c).

For large $A$, the stable dislocation has $g^{\prime}\left(u_{n}\right)>0$ for all $n$, and (2.6) gives 

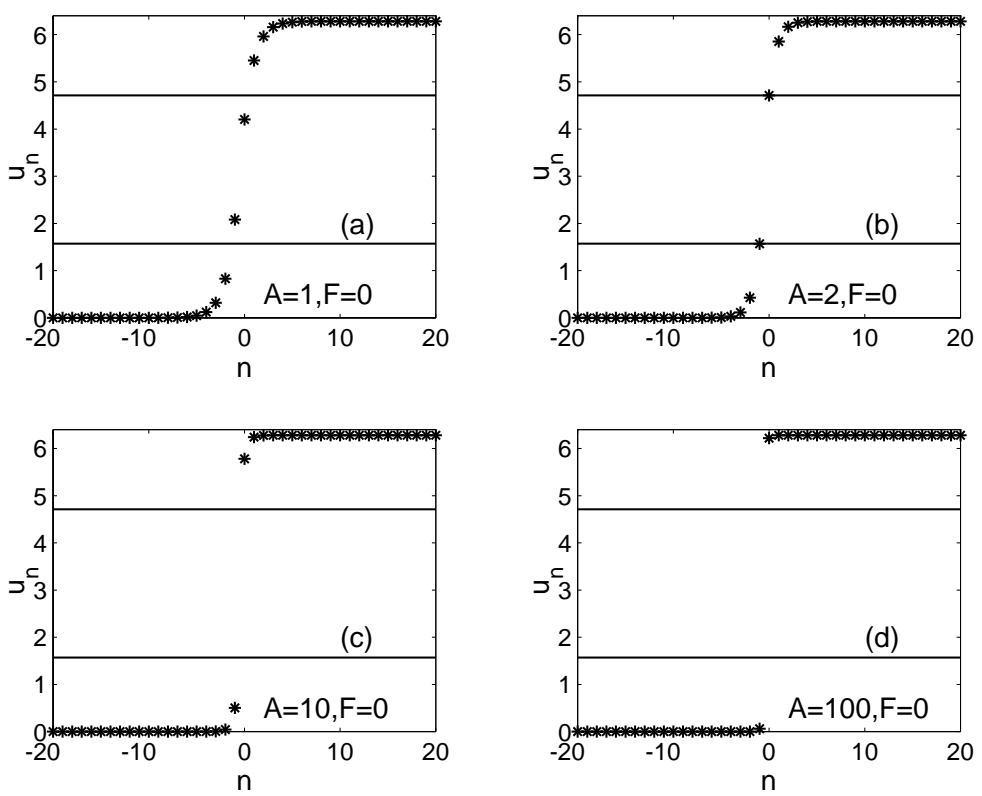

FIG. 2.4. Stationary solutions for the FK model when $A=1,2,10,100$.

$\lambda_{1}(A, 0)>0$. Since $\lambda_{1}\left(A, F_{c}\right)=0$, this implies that the critical field is nonzero. (Different proofs are given in [23,9].) As $A>0$ decreases, several $u_{n}$ may enter the region of negative slope $g^{\prime}(u)$ : the number of points with $g^{\prime}\left(u_{n}\right)<0$ increases as $A$ decreases; see Figures 2.2 and 2.4. It should then be possible to have $\lambda_{1}(A, 0)=0$, i.e., $F_{c}=0$, for a discrete system! Examples of this pinning anomaly will be given next.

2.3. Pinning failure. Despite widespread belief, it is not true that the critical field is positive for all discrete systems. This point was already raised by Hobart [21], who proposed the following numerical criterion to check whether for a given source $g$ the critical field for (1.1) is zero.

Let us assume for the sake of simplicity that $g$ is odd about 0 . Then $U_{2}(0)=0$ and $U_{1}(0)=-U_{3}(0)$. For any $x \in\left(U_{1}(0), U_{3}(0)\right)$, we can compute numerically a unique value $y(x)$ such that the sequence $u_{n}$ defined by $u_{0}=x, u_{1}=y(x)$, and $u_{n}=2 u_{n-1}-u_{n-2}+g\left(u_{n-1}\right), n>1$, tends to $U_{3}(0)$ as $n \rightarrow \infty$. Hobart conjectured that $F_{c}=0$ for a given nonlinearity $g$, provided that the function $y(x)$ satisfies

$$
y^{-1}(x)=-y(-x), \quad y(x)-y(-x)=2 x+g(x)
$$

for $x \in\left(U_{1}(0), U_{3}(0)\right)$. It is fairly easy to construct examples of nonlinearities $g(x)$ for which (2.7) holds. It suffices to choose some smooth odd increasing function $u(x)$ such that $u(x) \rightarrow \pm a$ as $x \rightarrow \pm \infty$ for some $a>0$. We define $g(u(x))=$ $u(x+1)-2 u(x)+u(x-1)$ so that $g(z)=u\left(u^{-1}(z)+1\right)-2 z+u\left(u^{-1}(z)-1\right)$ and $y(z)=u\left(u^{-1}(z)+1\right)$. Choosing $u(x)=\tanh (x)$ (see $[34,5,16]$ ), we get an explicit formula for $g: g(z)=-2 \gamma z\left(1-z^{2}\right) /\left(1-\gamma z^{2}\right)$ with $\gamma=\tanh ^{2}(1)$. Notice that one or two points of the stationary solutions, $u_{n}=\tanh (n+p)$ ( $p$ is any constant), enter the region where $g^{\prime}<0$; see Figure 2.5(a).

By following this procedure, we find examples of bistable source terms for which 

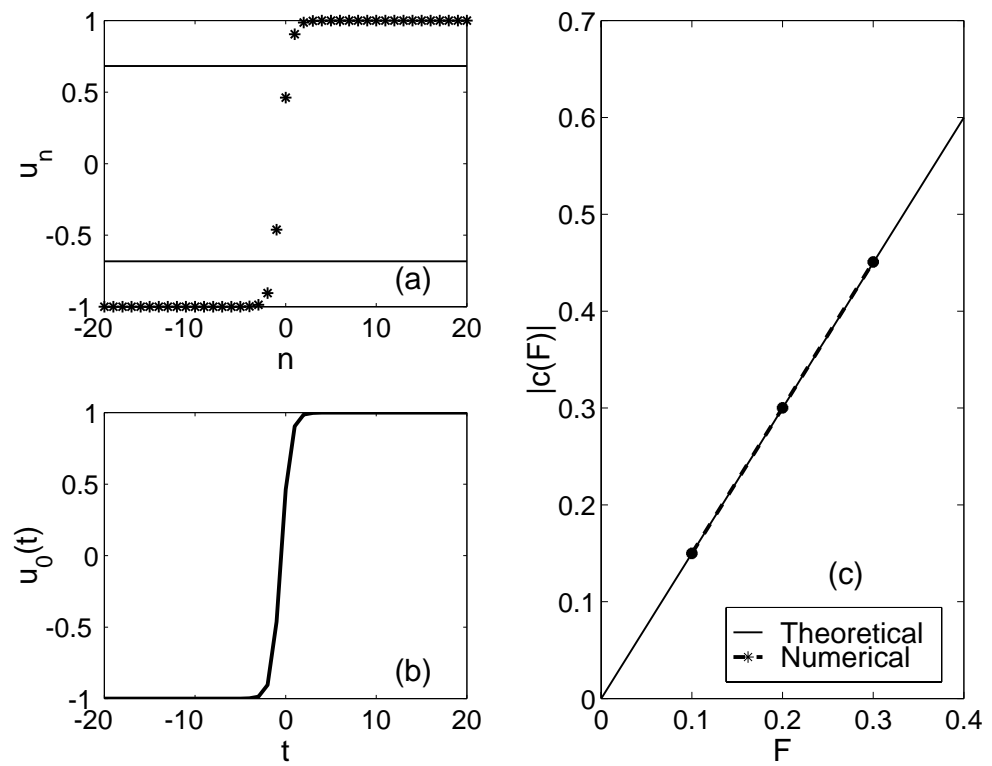

FIG. 2.5. (a) Stationary solution $u_{n}=\tanh (n)$, (b) wave front for $F$ small, (c) numerically calculated versus predicted speed for $g(u)=-2 \gamma u\left(1-u^{2}\right) /\left(1-\gamma u^{2}\right)$, with $\gamma=\tanh ^{2}(1)$.

(1.1) has a uniparametric family of continuous stationary solutions, $u_{n}=u(n+p)$, $0 \leq p<a$, satisfying $u_{n+1}-2 u_{n}+u_{n-1}=g\left(u_{n}\right)$ and $u_{-\infty}=-a, u_{\infty}=a$. In this case, (1.1) does not have stationary solutions joining $U_{1}(F / A)$ and $U_{3}(F / A)$ unless $F=0$ (see [8]). The existence of continuous steady solutions for $F=0$ implies that there is a continuous transition from wave fronts traveling to the left $(c<0)$ for $F>0$ to wave fronts traveling to the right $(c>0)$ for $F<0$. Only at $F=0$ are wave fronts stationary (pinned). This pinning anomaly is stated more precisely as follows.

TheOREM 2.1. Let $g \in C^{2}$ be as in the Introduction with $g(0)=0$, and let $\mathcal{L}(F)$ be the operator

$$
\mathcal{L}(F) v_{n}=A g^{\prime}\left(u_{n}\right) v_{n}+2 v_{n}-v_{n+1}-v_{n-1},
$$

corresponding to the evolution equation (1.1) linearized about the stationary solution $u_{n}=u_{n}(A, F)$ at field $F$. Let us assume that for $F=0$ there exists a differentiable increasing stationary solution $u(x)$ such that $u(x) \rightarrow \pm U_{3}(0)$ as $x \rightarrow \pm \infty$. Then,

1. zero is the smallest eigenvalue of the operator $\mathcal{L}_{0}=\mathcal{L}(0)$, corresponding to the evolution equation (1.1) linearized about the stationary solution $u_{n}(A, 0)=u(n)$;

2. $F_{c}(A)=0$ for $(1.1)$

3. traveling wave fronts exist for all $F \neq 0$. Furthermore, their speed increases linearly with the force for small $F$. We have

$$
c \sim-F \frac{U_{3}(0)-U_{1}(0)}{\int_{-\infty}^{\infty}\left(\frac{d u}{d x}\right)^{2} d x}
$$

as $F \rightarrow 0$.

Moreover, statement 3 implies the existence of steady differentiable solutions $u(x)$ of (1.1) such that $u(x) \rightarrow \pm U_{3}(0)$ as $x \rightarrow \pm \infty$ for $F=0$. 
It is not our goal here to give a rigorous proof of this result, but to sketch the main ideas. First of all, note that the derivative $v_{n}=u_{x}(n)>0$ is a positive eigenfunction of the elliptic operator $\mathcal{L}_{0}$ corresponding to the eigenvalue $\lambda=0$ and decaying exponentially at infinity. Statement 1 immediately follows. This fact can be used to construct propagating sub- and supersolutions for (1.1) which forbid pinning for any $F \neq 0$. Thus, $F_{c}=0$, which is statement 2 . For $F=\epsilon>0$ sufficiently small, the propagating subsolutions are $l_{n}(t)=l\left(n+\epsilon c_{0} t\right)$, with $c_{0}>0$ and $l(x)=u(x)+\epsilon u_{x}(x)$. For $F=-\epsilon$, the propagating supersolutions are $w_{n}(t)=w\left(n-\epsilon c_{0} t\right)$, with $c_{0}>0$ and $w(x)=u(x)-\epsilon u_{x}(x)$. In both cases, we have to choose $c_{0}<1 / \max \left(u_{x}\right)$. A subsolution traveling to the left "pushes" the fronts to the left. Similarly, the supersolutions traveling to the right "push" the fronts to the right.

Let us now obtain statement 3 . If $|F|>0$, we have traveling wave front solutions $u_{n}(t)=\mathcal{U}(n-c t)$ of $(1.1)$, whose profile $\mathcal{U}(z)$ satisfies the differential-difference equation

$$
-c \frac{d \mathcal{U}}{d z}(z)=\mathcal{U}(z+1)-2 \mathcal{U}(z)+\mathcal{U}(z-1)-g(\mathcal{U}(z))+F,
$$

and $\mathcal{U}( \pm \infty)= \pm U_{3}(0)$; see [8]. Let $F=F_{0} \epsilon$ with $0<\epsilon \ll 1$. The traveling wave solution can be written as $\mathcal{U}(n-c t)=u(n-c t)+\epsilon w(n-c t)+o(\epsilon)$, where $u(x)$ is the smooth stationary profile. Let $z=n-c t$ and $c=c_{0} \epsilon+o(\epsilon)$. Then $w$ obeys

$$
\begin{array}{r}
w(z+1)-2 w(z)+w(z-1)-A g^{\prime}(u(z)) w(z)=-c_{0} \frac{d u}{d z}(z)-F_{0}, \\
w(-\infty)=w(\infty)=\frac{1}{g^{\prime}\left(U_{1}\right)}=\frac{1}{g^{\prime}\left(U_{3}\right)} .
\end{array}
$$

By the Fredholm alternative, this linear nonhomogeneous equation has a solution if the left-hand side $-c_{0} d u / d z-F_{0}$ is orthogonal to the eigenfunction $d u / d z$, which yields (2.9).

In section 3, we show that the wave front speed $c$ scales as $\left|F-F_{c}\right|^{\frac{1}{2}}$ if $F_{c}>0$. Our linear scaling (2.9) of the velocity in statement 3 therefore implies that $F_{c}=0$. The linear scaling (2.9) with $F_{c}=0$ implies the existence of smooth stationary solutions at $F=0$ as discussed in the first subsection.

Remark 1. We conjecture that the three statements in Theorem 2.1 are equivalent. To prove this, it would be enough to show that $F_{c}=0$ implies the linear scaling of the speed of the waves (statement 3 ). Then, existence of differentiable stationary solutions follows. This implies statement $1\left(\lambda_{1}(A, 0)=0\right)$, which implies statement 2 $\left(F_{c}=0\right)$, as we showed above.

Remark 2. When stationary wave front solutions have smooth profiles, pinning failure occurs for discrete RD equations and for discrete equations with conservative dynamics. In the latter case, translation-invariant smooth profiles have the same energy, and therefore the PN energy barrier (defined as the smallest energy barrier that must be overcome for a kink or wave front to move [4]) vanishes. Pinning of a wave front usually results if the energy difference between the stable and the unstable front solutions (see Figure 2.2) is not zero. This energy difference provides an estimation of the PN energy barrier. Discussions of the PN potential and the PN barrier can be found in section 2.3 of [4] and in section III.B of [16]. The mathematical meaning and usefulness of the PN barrier for an infinite system with conservative dynamics are worth studying.

Remark 3. Speight and Ward [36] and Speight [37, 38] have developed a technique to discretize some continuum conservative models in such a way that kink-like initial 
profiles may propagate without getting trapped. Their idea is to seek a discrete version of the potential energy which admits minimals satisfying a first order difference equation called the Bogomol'nyi equation so that there is no PN barrier. On the other hand, the difference operators in Speight and Ward [36] discretized equations of motion have a structure different from discrete diffusion and are hard to justify physically.

Remark 4. In discrete RD equations, moving and pinned fronts cannot coexist for the same value of the applied field. For chains with conservative Hamiltonian dynamics, the situation is less clear. In fact, it is possible to have two stationary front solutions with a positive energy difference between them (which would imply a nonzero critical field and therefore wave front pinning according to general belief), and yet a moving wave front may coexist with the stationary fronts for the same parameter values. An explicit example of this situation has been constructed by Flach, Zolotaryuk, and Kladko using an inverse method [16].

3. Asymptotic theory of wave front depinning. In this section we introduce a systematic procedure for deriving analytic expressions for the critical field $F_{c}>0$ as a function of $A$, and for the front profiles and their velocity as functions of $F-F_{c}$ and $A$. Our methods work best in the strongly discrete case for large $A$. Our ideas are quite general and may be applied successfully to more complex discrete models [11]. We shall assume that $g \in C^{2}$ throughout this section.

3.1. Theory with a single active point. We choose $A$ large enough for the stable dislocation in Figure 2.2 not to enter the region where $g^{\prime}<0$; see Figure 2.4. When $F>0$, this solution is no longer symmetric with respect to $U_{2}$. If $F$ is not too large, all $u_{n}(A, F)$ avoid the region of negative slope $g^{\prime}(u)<0$. For larger $F$ and generic potentials $\left(\mathrm{FK}\right.$, double-well, ...), we have observed numerically that $g^{\prime}<0$ for a single point, labelled $u_{0}(A, F)$. This property persists until $F_{c}$ is reached; see Figure 3.1.
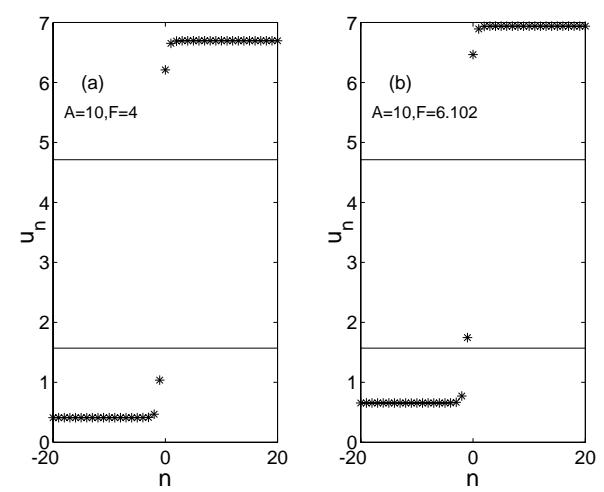

FIG. 3.1. Stationary solutions for the FK model with $A=10$ : (a) No points are found in the region $g^{\prime}<0$ for sufficiently small $F$; (b) one point enters the region $g^{\prime}<0$ for sufficiently large $F<F_{c}$.

First, consider the symmetric stationary profile with $u_{n} \neq U_{2}$ for $F=0$. The front profile consists of two tails with points very close to $U_{1}$ and $U_{3}$, plus two symmetric points $u_{0}, u_{1}$ in the gap region between $U_{1}$ and $U_{3}$. As $F>0$ increases, this profile changes slightly: the two tails are still very close to $U_{1}(F / A)$ and $U_{3}(F / A)$. As for the two middle points, $u_{1}$ gets closer and closer to $U_{3}$, whereas $u_{0}$ moves away from 

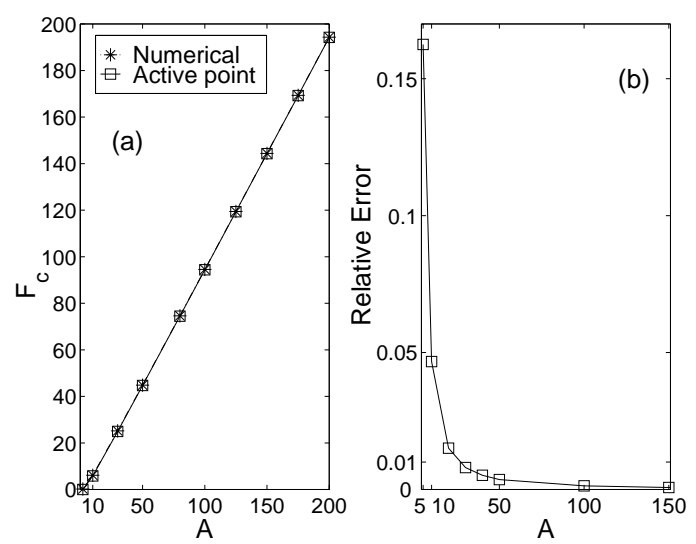

FIG. 3.2. Approximation of (1.1) by the equation with one active point for the FK potential and $A>2$ : (a) critical force versus $A$, (b) error in the approximation of $F_{c}(A)$.

$U_{1}$. This structure is preserved by the traveling fronts above the critical field: there is only one active point most of the time, which we can adopt as our $u_{0}$. Then the wave front profile $(2.2)$ can be calculated as $u(-c t)=u_{0}(t)$. In (1.1), we can approximate $u_{-1} \sim U_{1}, u_{1} \sim U_{3}$, thereby obtaining

$$
\frac{d u_{0}}{d t} \approx U_{1}\left(\frac{F}{A}\right)+U_{3}\left(\frac{F}{A}\right)-2 u_{0}-A g\left(u_{0}\right)+F .
$$

This equation has three stationary solutions for $F<F_{c}$, two stable and one unstable, and only one stable stationary solution for $F>F_{c}$. Let us consider $F<F_{c}$. Only two out of the three solutions of (3.1) approximate stationary fronts for the exact system: those having smaller values of $u_{0}$. The one having smallest $u_{0}$ approximates the stable stationary front; the other one approximates the unstable stationary front. Recall that the unstable front had a value $u_{0}=\left[U_{1}(0)+U_{3}(0)\right] / 2$ at the middle of the gap for $F=0$. As $F>0$ increases, $u_{0}$ decreases towards $U_{1}(F / A)$. Thus one active point will also approximate the profile of the unstable stationary front. The stationary solution of (3.1) having the largest value of $u_{0}$ (slightly below $U_{3}(F / A)$ ) is not consistent with the assumptions we made to derive (3.1), and therefore it does not approximate a physically existing stationary front. If $F>F_{c}$, the only stationary solution of (3.1) is the unphysical one. The critical field $F_{c}$ is such that the expansion of the right-hand side of (3.1) about the two coalescing stationary solutions has zero linear term, $2+A g^{\prime}\left(u_{0}\right)=0$, and

$$
2 u_{0}+A g\left(u_{0}\right) \sim U_{1}\left(\frac{F_{c}}{A}\right)+U_{3}\left(\frac{F_{c}}{A}\right)+F_{c} .
$$

These equations for $F_{c}$ and $u_{0}\left(A, F_{c}\right)$ have been solved for the FK potential, for which $u_{0}=\cos ^{-1}(-2 / A)$ and $U_{1}+U_{3}=2 \sin ^{-1}\left(F_{c} / A\right)+2 \pi$. The results are depicted in Figure 3.2 and show excellent agreement with those of direct numerical simulations for $A>10$. Our approximation performs less well for smaller $A$, and it breaks down at $A=2$ with the wrong prediction $F_{c}=0$. Notice that $F_{c}(A) / A \sim 1$ as $A$ increases. In practice, only steady solutions are observed for very large $A$.

Let us now construct the profile of the traveling wave fronts after depinning for 
$F$ slightly above $F_{c}$. Then $u_{0}(t)=u_{0}\left(A, F_{c}\right)+v_{0}(t)$ obeys the following equations:

$$
\begin{aligned}
\frac{d v_{0}}{d t} & =\alpha\left(F-F_{c}\right)+\beta v_{0}^{2}, \\
\alpha & =1+\frac{1}{A g^{\prime}\left(U_{1}\left(F_{c} / A\right)\right)}+\frac{1}{A g^{\prime}\left(U_{3}\left(F_{c} / A\right)\right)}, \\
\beta & =-\frac{A}{2} g^{\prime \prime}\left(u_{0}\right),
\end{aligned}
$$

where we have used $2+A g^{\prime}\left(u_{0}\right)=0$ and (3.2) and ignored terms of order $\left(F-F_{c}\right) v_{0}$ and higher. These terms are negligible after rescaling $v_{0}=\left(F-F_{c}\right)^{\frac{1}{2}} \varphi$ and $\tau=$ $\left(F-F_{c}\right)^{\frac{1}{2}} t$. The coefficients $\alpha$ and $\beta$ are positive because $g^{\prime}\left(U_{i}\right)>0$ for $i=1,3$ and $g^{\prime \prime}\left(u_{0}\right)<0$ since $u_{0} \in\left(U_{1}(0), U_{2}(0)\right)$. For the FK potential, $\alpha=1+2 / \sqrt{A^{2}-F_{c}^{2}}$ and $\beta=\sqrt{A^{2}-F_{c}^{2}} / 2$. Equation (3.3) has the (outer) solution

$$
v_{0}(t) \sim \sqrt{\frac{\alpha\left(F-F_{c}\right)}{\beta}} \tan \left(\sqrt{\alpha \beta\left(F-F_{c}\right)}\left(t-t_{0}\right)\right),
$$

which is very small most of the time, but it blows up when the argument of the tangent function approaches $\pm \pi / 2$. Thus the outer approximation holds over a time interval $\left(t-t_{0}\right) \sim \pi / \sqrt{\alpha \beta\left(F-F_{c}\right)}$, which equals $\pi \sqrt{2 / \alpha}\left(A^{2}-4\right)^{-\frac{1}{4}}\left(F-F_{c}\right)^{-\frac{1}{2}}$ for the FK potential. The reciprocal of this time interval yields an approximation for the wave front velocity,

$$
c(A, F) \sim-\frac{\sqrt{\alpha \beta\left(F-F_{c}\right)}}{\pi},
$$

or $c \sim-\left(A^{2}-4\right)^{\frac{1}{4}}\left(1+2 / \sqrt{A^{2}-F_{c}^{2}}\right)^{\frac{1}{2}}\left(F-F_{c}\right)^{\frac{1}{2}} /(\pi \sqrt{2})$ for an FK potential. The minus sign reminds us that wave fronts move towards the left for $F>F_{c}$. In Figures $3.3(\mathrm{a})$ and (b) we compare this approximation with the numerically computed velocity for $A=100$ and $A=10$.

When the solution begins to blow up, the outer solution (3.6) is no longer a good approximation, for $u_{0}(t)$ departs from the stationary value $u_{0}\left(A, F_{c}\right)$. We must go back to (3.1) and obtain an inner approximation to this equation. As $F$ is close to $F_{c}$ and $u_{0}(t)-u_{0}\left(A, F_{c}\right)$ is of order 1 , we numerically solve $(3.1)$ at $F=F_{c}$ with the matching condition that $u_{0}(t)-u_{0}\left(A, F_{c}\right) \sim 2 /\left[\pi \sqrt{\beta /\left[\alpha\left(F-F_{c}\right)\right]}-2 \beta\left(t-t_{0}\right)\right]$ as $\left(t-t_{0}\right) \rightarrow-\infty$. This inner solution describes the jump of $u_{0}$ from $u_{0}\left(A, F_{c}\right)$ to values on the largest stationary solution of (3.1), which is close to $U_{3}$. During this jump, the motion of $u_{0}$ forces the other points to move. Thus, $u_{-1}(t)$ can be calculated by using the inner solution in (1.1) for $u_{0}$, with $F=F_{c}$ and $u_{-2} \approx U_{1}$. A composite expansion [2] constructed with these inner and outer solutions is compared to the result of direct numerical simulations in Figure 3.4.

Notice that (3.3) is the normal form associated with a saddle-node bifurcation in a one-dimensional phase space. The wave front depinning transition is a global bifurcation with generic features: each individual point $u_{n}(t)$ spends a long time, which scales as $\left|F-F_{c}\right|^{-\frac{1}{2}}$, near discrete values $u_{n}\left(A, F_{c}\right)$, and then jumps to the next discrete value on a time scale of order 1 . The traveling wave ceases to exist for $F \leq F_{c}$.

3.2. Theory with several active points. The approximations to $F_{c}(A)$ and the wave front speed provided by the previous asymptotic theory break down for small 

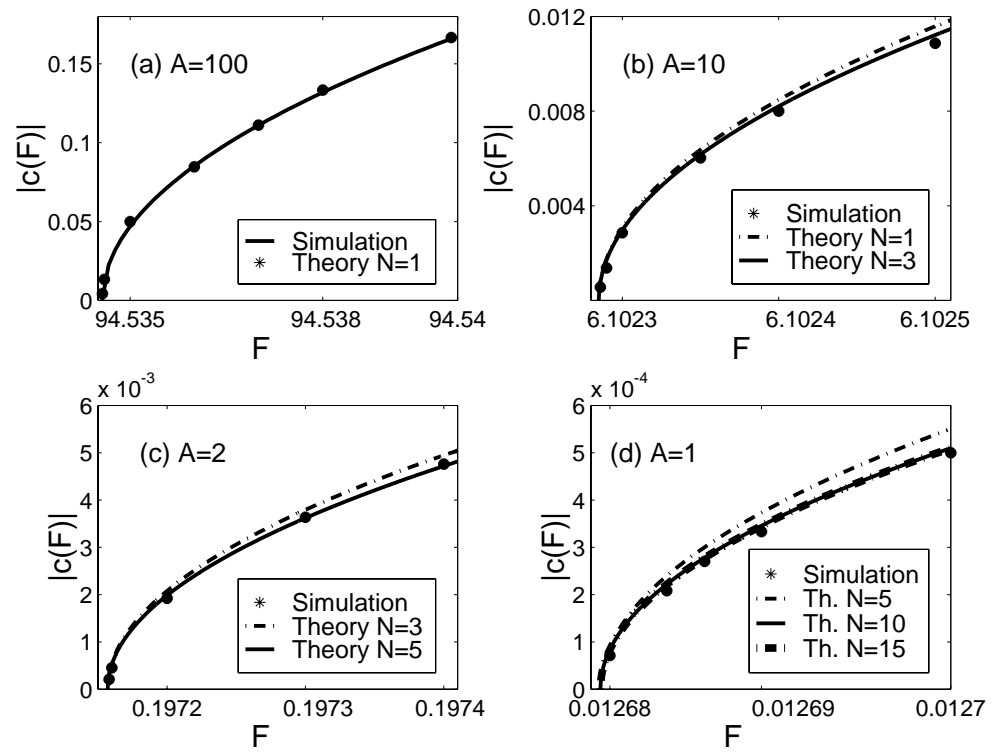

FIG. 3.3. Comparison of theoretically predicted and numerically calculated wave front velocities near $F_{c}$ for the FK model with $N$ active points and the following values of the parameter A: (a) $A=100$, (b) $A=10$, (c) $A=2$, (d) $A=1$.
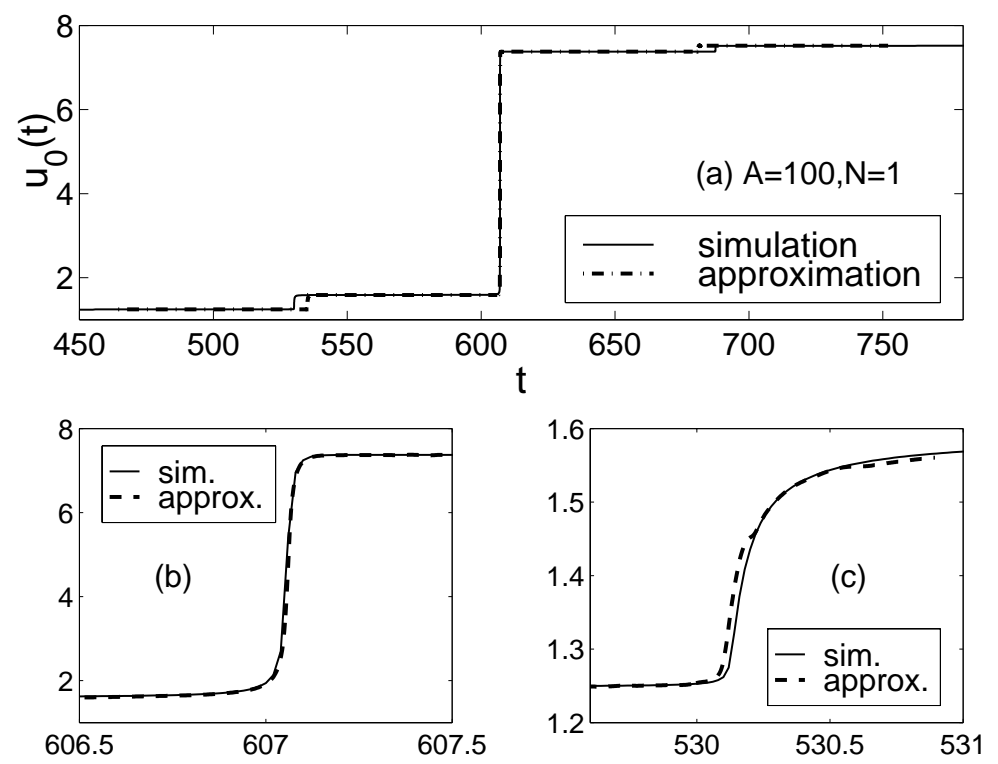

FIG. 3.4. Comparison of asymptotic and numerically calculated wave front profiles near $F_{c}$ : (a) Complete wave front profile as indicated by the trajectory $u_{0}(t)$. (b) Zoom near the largest jump in the profile. (c) Zoom near the jump preceding the largest one after translating the asymptotic profile. This last has been calculated by inserting the approximate $u_{0}(t)$ in the equation for $u_{-1}(t)$.

$A$. In particular, for the FK potential and $A<2$, no double zeroes of $2 x+A \sin (x)-$ $\left(F+U_{1}+U_{3}\right)$ are found for $F=F_{c}$. What happens is that we need more than one point to approximate wave front motion. Depinning is then described by a reduced 
system of more than one degree of freedom corresponding to active points. There is a saddle-node bifurcation in this reduced system whose normal form is of the same type as (3.3). The jump of the active points after blow up is found by solving the reduced system with a matching condition [10].

We explain our procedure for a finite number of active points. The front is formed by two tails, very close to $U_{1}(F / A)$ and $U_{3}(F / A)$, respectively, and several intermediate points $u_{-L}, \ldots, u_{M}$. The reduced system describing the dynamics of the front for $F>F_{c}$ is

$$
\begin{array}{r}
\frac{d u_{i}}{d t}=u_{i+1}-2 u_{i}+u_{i-1}-A g\left(u_{i}\right)+F, \quad i=-L, \ldots, M, \\
u_{-L-1}=U_{1}(F / A), \quad u_{M+1}=U_{3}(F / A) .
\end{array}
$$

At $F=F_{c}$ this system has a stationary solution $u_{-L}, \ldots, u_{M}$. Writing $u_{i}(t)=u_{i}+$ $v_{i}(t)$, we obtain for $v_{i}$

$$
\begin{aligned}
\frac{d v_{i}}{d t}=v_{i+1}-2 v_{i}+v_{i-1}-A g^{\prime}\left(u_{i}\right) v_{i}-\frac{A}{2} g^{\prime \prime}\left(u_{i}\right) v_{i}^{2}+F-F_{c}, & i=-L, \ldots, M, \\
v_{-L-1} & \sim \frac{F-F_{c}}{A g^{\prime}\left(U_{1}\left(F_{c} / A\right)\right)}, \quad v_{M+1} \sim \frac{F-F_{c}}{A g^{\prime}\left(U_{3}\left(F_{c} / A\right)\right)} .
\end{aligned}
$$

The tridiagonal matrix $\mathcal{M}$ defined as

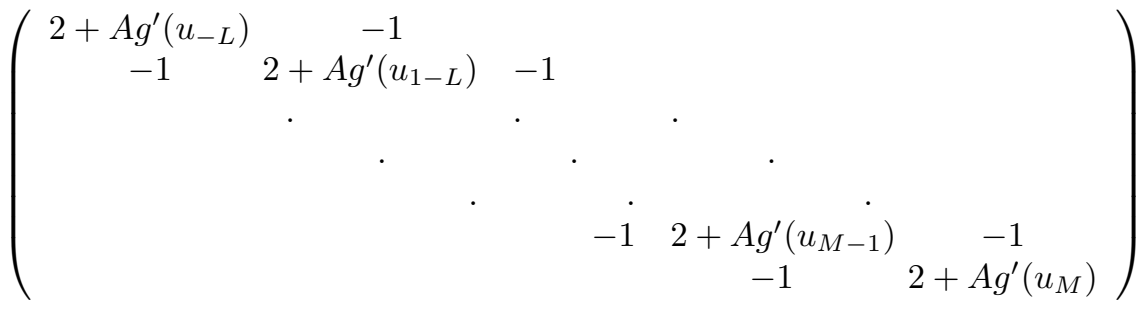

has $L+M$ strictly positive eigenvalues plus a smallest eigenvalue $\lambda \sim 0$ with an associate positive eigenfunction $V$. We choose $V$ such that $\sum_{i=-L}^{M} V_{i}^{2}=1$ and write an eigenfunction expansion for $v$ as follows:

$$
v(t)=\varphi V+\sum_{i=-L, i \neq 0}^{M} W_{i} \varphi_{i} \exp \left(-\lambda_{i} t\right) .
$$

Thus $v(t) \sim V \varphi(t)$ as time increases. Let $D=-\frac{A}{2} \operatorname{diag}\left(g^{\prime \prime}\left(u_{-L}\right), \ldots, g^{\prime \prime}\left(u_{M}\right)\right)$, $V^{2}=\left(V_{-L}^{2}, \ldots, V_{M}^{2}\right)$, and

$$
w=\left(F-F_{c}\right)\left(1+\frac{1}{A g^{\prime}\left(U_{1}\left(F_{c} / A\right)\right)}, 1, \ldots, 1,1+\frac{1}{A g^{\prime}\left(U_{3}\left(F_{c} / A\right)\right)}\right) .
$$

Then system (3.9) becomes

$$
\frac{d \varphi}{d t} V \sim \varphi \mathcal{M} V+\varphi^{2} D V^{2}+w \sim \varphi^{2} D V^{2}+w
$$

as time increases. Multiplying by the transpose of $V$, we get an evolution equation for the amplitude $\varphi(t)$ :

$$
\frac{d \varphi}{d t}=\alpha\left(F-F_{c}\right)+\beta \varphi^{2}
$$


where now $\alpha$ and $\beta$ are

$$
\begin{array}{r}
\alpha=\sum_{i=-L}^{M} V_{i}+\frac{V_{-L}}{A g^{\prime}\left(U_{1}\left(F_{c} / A\right)\right)}+\frac{V_{M}}{A g^{\prime}\left(U_{3}\left(F_{c} / A\right)\right)}>0, \\
\beta=-\frac{A}{2} \sum_{i=-L}^{M} g^{\prime \prime}\left(u_{i}\right) V_{i}^{3}>0 .
\end{array}
$$

The coefficient $\alpha$ is positive because $g^{\prime}\left(U_{i}\right)>0$ for $i=1,3$. We have checked numerically that $\beta>0$ for different nonlinearities and values of $A$. An intuitive explanation follows. First, notice that $g^{\prime \prime}(u)>0$ for $u \in\left(U_{2}(0), U_{3}(0)\right)$, and $g^{\prime \prime}(u)<0$ for $u \in\left(U_{1}(0), U_{2}(0)\right)$. For large $A$, the largest component is $V_{0}$, the others are negligible, and we have one active point as in the previous subsection; see Figure 3.4. Then $\beta \sim-g^{\prime \prime}\left(u_{0}\left(A, F_{c}\right)\right) V_{0}^{3}>0$ because $u_{0}<U_{2}(0)$, which implies $g^{\prime \prime}\left(u_{0}\right)<0$. As $A$ decreases, $V_{0}$ is still the largest component and $g^{\prime \prime}\left(u_{0}\left(A, F_{c}\right)\right)<0$. Now there may be other terms with $g^{\prime \prime}\left(u_{i}\left(A, F_{c}\right)\right)>0$, and we have only numerical evidence that $\beta>0$, not a proof.

Notice that (3.13) is the normal form of a saddle-node bifurcation. Its solution is again (3.6), which blows up at times $\left(t-t_{0}\right)= \pm 1 /(2 c)$, where

$$
c(A, F) \sim-\frac{1}{\pi} \sqrt{\alpha \beta\left(F-F_{c}\right)},
$$

as discussed before. $c$ is the wave front speed near $F_{c}$, approximately given by the reciprocal of the time during which the outer solution holds.

Figures 2.1, 3.3, and 3.5 show the critical field, wave front velocities, and profiles for different values of $A \in(1,10)$ corresponding to the FK model. We have compared results of direct numerical simulations to those of our theory for $N=L+M+1$ active points. Provided that $N=L+M+1$ active points have been selected, we find the smallest eigenvalue of the matrix $\mathcal{M}$ and move $F$ until $\lambda(F, A ; N)=0, N=L+M+1$, which yields an approximation for $F_{c}(A)$; see Figure 2.1. The wave front velocities can be calculated by means of (3.14) and have been depicted in Figure 3.3.

The wave front profiles near $F_{c}$ can be determined as follows. We start with an initial condition, $u_{n}(0) \approx u_{n}\left(A, F_{c}\right)$ or $\varphi(0)=0$ in (3.11). The active points blow up at $t \sim \pm(2 c)^{-1}$, for example as

$$
u_{n}(t) \sim u_{n}\left(A, F_{c}\right)+\frac{1}{\beta\left( \pm \frac{1}{2 c}-t\right)} V,
$$

provided $t \rightarrow \pm 1 /(2 c)$. At these times, we should insert a fast stage during which the $u_{n}(t)$ are no longer close to $u_{n}\left(A, F_{c}\right)$, as an inner layer. The inner layer variables $u_{n}(t)$ obey (1.1) with $F=F_{c}$ and the boundary conditions $u_{n}(t) \rightarrow u_{n}\left(A, F_{c}\right)$ (according to $(3.15))$ as $t \rightarrow-\infty$, and $u_{n}(t) \rightarrow u_{n+1}\left(A, F_{c}\right)$ as $t \rightarrow \infty$. To get a uniform approximation, we notice that the blow up times are $t_{m}=(2 c)^{-1}+m / c, m \in \mathbb{Z}$. Let us denote by $u_{n}^{(m)}(\tau), \tau=\left(t-t_{m}\right)$, the solution of (1.1) with $F=F_{c}$ and the boundary conditions $u_{n}^{(m)}(\tau) \rightarrow u_{n+m}\left(A, F_{c}\right)$ as $\tau \rightarrow-\infty$, and $u_{n}^{(m)}(\tau) \rightarrow u_{n+m+1}\left(A, F_{c}\right)$ as $\tau \rightarrow$ $\infty$. During the time interval $\left(t_{-L-n-1}, t_{M-n}\right)=\left(-(2 c)^{-1}-(L+n) / c,(2 c)^{-1}+(M-\right.$ $n) / c$ ) that $u_{n}(t)$ needs to go from $U_{1}\left(F_{c} / A\right)$ to $U_{3}\left(F_{c} / A\right)$, the uniform approximation 

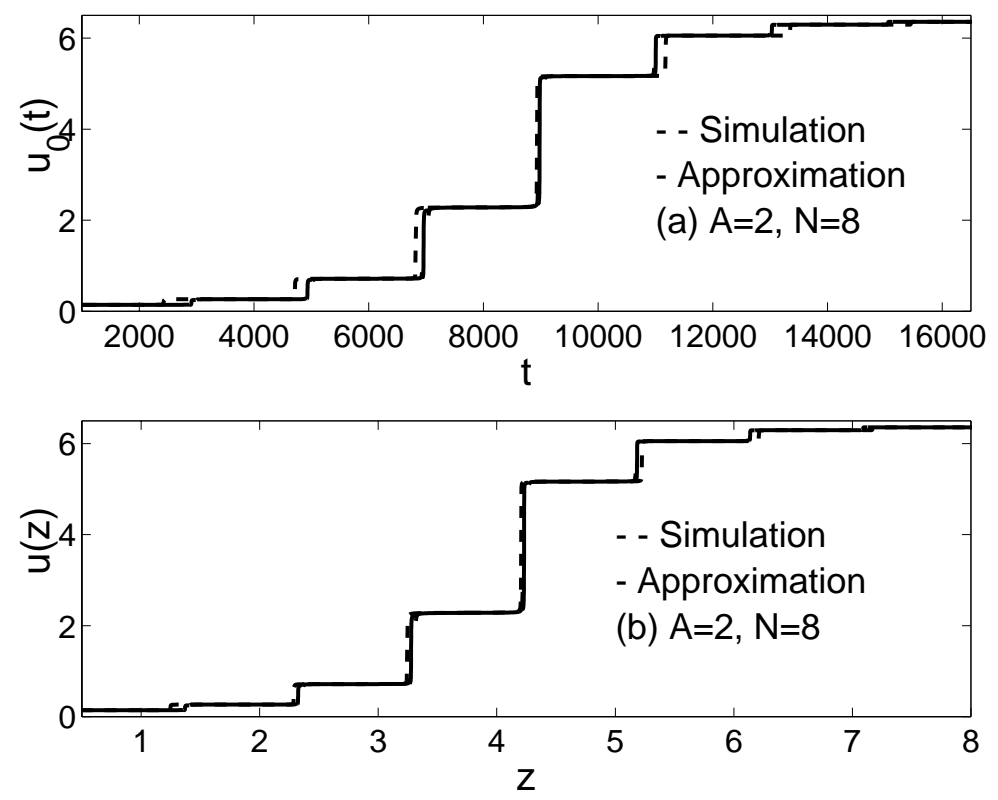

FiG. 3.5. Comparison of theoretically predicted and numerically calculated wave fronts near $F_{c}$ for $A=2$ using $N=8$ active points: (a) trajectory of one point, (b) wave front profile, $u(z)=$ $u_{0}(z /|c|)$.

to the wave front is

$$
\begin{aligned}
& u_{n}(t) \sim \sum_{m=-n-L-1}^{M-n}\left\{u_{n}^{(m)}\left(t-t_{m}\right)+u_{n}^{(m-1)}\left(t-t_{m-1}\right)-u_{n+m}\left(A, F_{c}\right)\right. \\
&+ {\left.\left[\varphi\left(t-\frac{m}{c}\right)-\frac{1}{\beta\left(t_{m}-t\right)}+\frac{1}{\beta\left(t-t_{m-1}\right)}\right] V\right\} \chi_{\left(t_{m-1}, t_{m}\right)} . }
\end{aligned}
$$

Then $u_{n}\left(t_{-L-n-1}\right) \sim U_{1}\left(F_{c} / A\right)$ and $u_{n}\left(t_{M-n}\right) \sim U_{3}\left(F_{c} / A\right)$. In (3.16), the indicator function $\chi_{\left(t_{m-1}, t_{m}\right)}$ is 1 if $t_{m-1}<t<t_{m}$ and 0 otherwise. Therefore, $\chi_{\left(t_{m-1}, t_{m}\right)}=$ $\theta\left(t_{m}-t\right)-\theta\left(t_{m-1}-t\right)$, where $\theta(x)=1$ if $x>0$ and 0 otherwise. Written in terms of the variable $z=n-c t$ such that $u_{n}(t)=u(z), u(z)=u_{n}((n-z) / c)=u_{0}(-z / c)$. Then (3.16) becomes

$$
\begin{aligned}
u(z) \sim \sum_{m=-L-1}^{M}\left\{u_{0}^{(m)}\left(-\frac{z+m+\frac{1}{2}}{c}\right)\right. & +u_{0}^{(m-1)}\left(-\frac{z+m-\frac{1}{2}}{c}\right)-u_{m}\left(A, F_{c}\right) \\
+\left[\varphi\left(-\frac{z+m}{c}\right)\right. & \left.\left.-\frac{c}{\beta\left(z+m+\frac{1}{2}\right)}-\frac{c}{\beta\left(z+m-\frac{1}{2}\right)}\right] V\right\} \\
& \times\left[\theta\left(z+m+\frac{1}{2}\right)-\theta\left(z+m-\frac{1}{2}\right)\right]
\end{aligned}
$$

for $-M-1 / 2<z<L+1 / 2$. We have $u(L+1 / 2+0) \sim U_{1}\left(F_{c} / A\right)$ and $u(-M-$ $1 / 2-0) \sim U_{3}\left(F_{c} / A\right)$, and therefore (3.17) approximates the wave front profile. In Figure 3.5 , we have depicted the wave front profile in two ways, by drawing $u_{0}(t)$ and $u(z)=u_{0}(-z / c)$. Notice that the largest source of discrepancy between numerical 
calculations and our asymptotic approximation is the error in determining the wave speed. The discrepancies are more evident for $u_{0}(t)$ because of the different horizontal scale used to depict $u(z)$.

How do we determine the optimal number of active points? For large enough $N=L+M+1$ and a given $A$, the eigenvector $V$ corresponding to the smallest eigenvalue of the matrix $\mathcal{M}$ in (3.10) has a certain number of components that are of order one, whereas all others are very small. The number of components of normal size determines the optimal number of active points: only one point if $A$ is larger than 10, five if $A=2$, etc. Keeping less active points than the optimal number results in larger errors, whereas keeping more active points than optimal does not result in a significantly better approximation. The eigenvector of the reduced system of equations for the active points is a good approximation to the large components of the eigenvector corresponding to the complete system. As we approach the continuum limit, more and more points enter the reduced system of equations, and exponential asymptotic methods become a viable alternative to our methods.

3.3. Depinning transition as a global bifurcation. We have shown that the depinning transition is a global bifurcation in a reduced system of equations corresponding to the active points. Starting from a stable stationary solution, the smallest eigenvalue of the system linearized about the stationary solution becomes zero at the (approximate) critical field, and its associated eigenfunction is positive. The stationary solution disappears as the critical field is surpassed. Beyond it, the active points $u_{n}(t)$ spend a long time, of order $\left(F-F_{c}\right)^{-\frac{1}{2}}$, near the stationary values $u_{n}\left(A, F_{c}\right)$, and then jump to $u_{n+1}\left(A, F_{c}\right)$ on an order 1 time scale. Near the critical field, the depinning transition is described locally by the normal form of a saddle-node bifurcation. For $F>F_{c}$ (or $F<-F_{c}$ ), the bifurcation amplitude blows up in finite time, on a time scale of order ||$F\left|-F_{c}\right|^{-\frac{1}{2}}$. The construction of the wave front profile is completed, matching the outer solution given by the saddle-node normal form to a solution of the reduced system of active points at $F=F_{c}$. (A mathematically related phenomenon occurs in a mean-field model of sliding charge-density waves [2].)

We conjecture that the depinning transition in the infinite system (1.1) is a global bifurcation of the same type as for the reduced system of active points. At the critical field, two stationary solutions of (1.1) (one stable, the other unstable) coalesce and disappear. For $F>F_{c}$ (or $F<-F_{c}$ ), the wave front profile is constructed as indicated above for the reduced system. To prove this conjecture, we could repeat our construction in section 3.2 for an infinite number of points. This is possible because we know that the infinite system, linearized about the "stable" steady solution $u_{i}\left(A, F_{c}\right)$ at $F=F_{c}$, has a zero eigenvalue and an associated positive exponentially decaying eigenfunction $V$. Using $V$, we obtain the normal form equation (3.13), where now

$$
\alpha=\sum_{i=-\infty}^{\infty} V_{i}>0, \quad \beta=-\frac{A}{2} \sum_{i=-\infty}^{\infty} g^{\prime \prime}\left(u_{i}\right) V_{i}^{3} .
$$

We should now prove that the coefficient $\beta$ is positive and that the infinite system has solutions connecting $u_{n}\left(A, F_{c}\right)$ to $u_{n+1}\left(A, F_{c}\right)$ and satisfying the matching condition. We justified that $\beta>0$ for the finite system in subsection 3.2, and we show in Proposition A.4 (in the appendix) that the eigenfunction for the infinite system can be approximated by the corresponding eigenfunction of the reduced system with a finite number of active points. The existence of traveling wave solutions for $F>F_{c}$ ensures that the infinite system has solutions connecting $u_{n}\left(A, F_{c}\right)$ to $u_{n+1}\left(A, F_{c}\right)$. 
The velocity of a wave front in the infinite system is again given by (3.14) with the coefficients (3.18).

Remark 5. By using comparison techniques, it is possible to prove that solutions of discrete RD equations with finitely many points and Dirichlet boundary conditions approximate solutions of the same equations with infinitely many points. In the continuum limit, the wave fronts approach constant values exponentially fast as $i \rightarrow$ $\pm \infty$. This exponential decay justifies the active point approximation in two ways. First, the number of active points needed to approximate well the wave fronts of the infinite system decreases as $A$ increases. It is usually better to add another active point to the approximate system than to patch rigid tails to the last active points of a wave front by generalizing Kladko, Mitkov, and Bishop's active site approximation [28]. Secondly, exponential decay at the ends of a wave front causes the operator of the linearized problem about the wave front to be compact and therefore to have a discrete spectrum (see the appendix). This fact justifies that the normal form we calculate by using active points approximates the correct local normal form of the depinning global bifurcation.

4. Conclusions. In this paper, we have studied depinning of wave fronts in discrete RD equations. Pinned (stationary) and traveling wave fronts cannot coexist for the same value of the forcing term. There are two different depinning transitions, i.e., two different ways in which a pinned front may start moving. The normal depinning transition can be viewed as a loss of continuity of traveling front profiles as the critical field is approached: below the critical field, the fronts become pinned stationary profiles with discontinuous jumps at discrete values $u_{n}$. The wave front velocity scales as $\left|F-F_{c}\right|^{\frac{1}{2}}$ near the critical field $F_{c}$. For sufficiently large $A$ (far from the continuum limit), the critical field and these fronts can be approximated by singular perturbation methods which show excellent agreement with numerical simulations. These methods are based upon the fact that the wave front motion can be described by a reduced system of equations corresponding to the dynamics of only a finite number of points, the active points.

Besides the normal depinning transition, certain nonlinearities present anomalous pinning (pinning failure): the velocity of the wave fronts is not zero except at zero forcing, just as for continuous RD equations. These nonlinearities are characterized by smooth profiles of stationary and moving wave fronts, by having zero critical field, and by a linear scaling of wave front velocity with field.

Appendix. Characterization of the depinning threshold. In this section we establish the "depinning criterion," which provides a characterization of $F_{c}(A)$ as follows.

Theorem A.1. Set $F=0$ and $A>0$. Assume that the nonlinearity $g \in C^{3}$ has three zeroes $U_{i}, U_{1}<U_{2}<U_{3}$, is odd about $U_{2}$, and satisfies $g^{\prime}\left(U_{1}\right)=g^{\prime}\left(U_{3}\right)>0$. Let $u_{n}$ be a stationary increasing solution of (1.1), symmetric about $U_{2}$ and such that $u_{-\infty}=U_{1}$ and $u_{\infty}=U_{3}$. Let $\lambda_{1}(A, 0)$ be the smallest eigenvalue of the zero field operator $\mathcal{L}_{0}$ of $(2.8)$ at $F=0$ :

$$
\begin{array}{r}
-\left(v_{n+1}-2 v_{n}+v_{n-1}\right)+A g^{\prime}\left(u_{n}\right) v_{n}=\lambda_{1}(A, 0) v_{n}, \\
v_{ \pm n} \rightarrow 0 \text { exponentially as } n \rightarrow \infty .
\end{array}
$$

If $\lambda_{1}(A, 0)>0$, then $F_{c}(A)>0$, and for $|F| \leq F_{c}(A)$ there exist increasing stationary solutions $u_{n}(A, F)$ of (1.1) with $u_{-\infty}=U_{1}(F / A)$ and $u_{\infty}=U_{3}(F / A)$. Moreover, the smallest eigenvalues of the operator $\mathcal{L}(F)$, corresponding to the linearization of (1.1) 
about $u_{n}(A, F)$,

$$
\begin{array}{r}
-\left(v_{n+1}-2 v_{n}+v_{n-1}\right)+A g^{\prime}\left(u_{n}(A, F)\right) v_{n}=\lambda_{1}(A, F) v_{n}, \\
v_{ \pm n} \rightarrow 0 \text { exponentially as } n \rightarrow \infty,
\end{array}
$$

are strictly positive for $|F|<F_{c}(A)$. We can characterize $F_{c}(A)$ as the zero of the smallest eigenvalue, $\lambda_{1}\left(A, F_{c}(A)\right)=0$.

This theorem will be proved in subsection A.2. To calculate $\lambda_{1}(A, F)$ and $F_{c}(A)$, we approximate the infinite tridiagonal matrix in (A.2) by an $N \times N$ matrix, where $N$ is the number of active points. Similar truncation approximations were used in [29] to calculate the lowest eigenvalue of an infinite tridiagonal matrix. For values of $A$ which are not too small, numerical simulations show that the matrices $\mathcal{M}$ in (3.10) have positive eigenvalues. The eigenvector $V(A, F, N)$ (chosen to have norm 1), corresponding to the smallest eigenvalue $\lambda(A, F, N)$, is positive, and it is "concentrated" in the central components $V_{-m(A)}, \ldots, V_{m(A)}$. All other components are very small. The number of significant components $m(A)$ does not change as $N$ increases, but it increases as $A$ decreases. For large $A, m(A)=0$, and only $V_{0}$ is significant. Provided $N$ is large enough, the eigenvalues $\lambda(A, F, N)$ and the eigenvectors $V(A, F, N)$ approximate well the smallest eigenvalue and associated eigenfunction of the infinite problem, as we indicate in the next subsection. For a fixed value of $N$, the eigenvalues $\lambda(A, F, N)$ decrease as $A$ decreases. For fixed $N$ and $A$, they decrease as $F$ increases from $F=0$ to values close to $F_{c}(A)$. In the next subsection, we collect several results on eigenvalues for this type of problem.

A.1. Eigenvalue problems. Before proving Theorem A.1, we should make sure that our linear operators do have eigenfunctions and eigenvalues. We consider the real valued and symmetric operators $\mathcal{L}(F) v_{n}=A g^{\prime}\left(u_{n}\right) v_{n}-\left(v_{n+1}-2 v_{n}+v_{n-1}\right)$ in spaces of sequences decaying exponentially at infinity. Their spectra are discrete and real (these operators are compact), and we would like to make sure that they are not empty. Since we are interested mainly in the smallest eigenvalue, we shall use its variational characterization, prove that this eigenvalue exists, and characterize its dependence on the parameters $A$ and $F$. We shall also describe finite-dimensional approximations of eigenvalues and eigenfunctions.

Let us first look for necessary conditions for $\lambda(A, F)$ to exist. Let $\lambda \in \mathbb{R}$ be an eigenvalue of $\mathcal{L}(F)$ with eigenfunction $V_{n}$. Multiplying $\mathcal{L}(F) V_{n}-\lambda V_{n}$ by $V_{n}$ and summing over $n$, we obtain

$$
\begin{aligned}
0 & =\sum_{n}\left(V_{n+1}-V_{n}\right)^{2}+\left[A g^{\prime}\left(u_{n}\right)-\lambda\right] V_{n}^{2} \\
& \geq \sum_{n}\left(V_{n+1}-V_{n}\right)^{2}+\left(\left[A \min _{n} g^{\prime}\left(u_{n}\right)\right]-\lambda\right) \sum_{n} V_{n}^{2} .
\end{aligned}
$$

Thus, $\lambda=\sum_{n}\left[\left(V_{n+1}-V_{n}\right)^{2}+A g^{\prime}\left(u_{n}\right) V_{n}^{2}\right] / \sum V_{n}^{2}>A \min _{n} g^{\prime}\left(u_{n}\right)$. This inequality implies that $\lambda$ is positive if $u_{n}$ does not take on values in the region where $g^{\prime}$ is negative, which occurs for large enough $A>0$. In general, we can say only that $\lambda>A g^{\prime}\left(U_{2}\right)$ for $g^{\prime}$ attains its minimum value in $\left[U_{1}, U_{3}\right]$ at $U_{2}$. Similarly,

$$
\begin{aligned}
0 & =\sum_{n}\left(V_{n+1}-V_{n}\right)^{2}+\left[A g^{\prime}\left(u_{n}\right)-\lambda\right] V_{n}^{2} \\
& \leq\left[4+A \max _{n}\left(g^{\prime}\left(u_{n}\right)\right)-\lambda\right] \sum_{n} V_{n}^{2} .
\end{aligned}
$$


Therefore, $\lambda<A g^{\prime}\left(U_{1}\right)+4=A g^{\prime}\left(U_{3}\right)+4$ for $g^{\prime}(u)$ attains its maximum value in $\left[U_{1}, U_{3}\right]$ at the end points, $u=U_{1}$ and $u=U_{3}$.

The smallest eigenvalue $\lambda$ is given by the Rayleigh formula

$$
\lambda=\min \frac{\sum_{n}\left(w_{n+1}-w_{n}\right)^{2}+A g^{\prime}\left(u_{n}\right) w_{n}^{2}}{\sum_{n} w_{n}^{2}},
$$

where the infimum is taken over our space of exponentially decaying functions. That minimum is attained at an eigenfunction $V_{n}$ solving (A.1). Now, (A.1) may have solutions decaying at $\pm \infty$ only if the difference equation

$$
V_{n+1}+\left(-2-A g^{\prime}\left(U_{1}\right)+\lambda\right) V_{n}+V_{n-1}=0
$$

has solutions of the form $r^{n}$ with $r<1$. This happens when $\left(-2-A g^{\prime}\left(U_{1}\right)+\lambda\right)^{2}>4$. Thus either $\lambda>4+A g^{\prime}\left(U_{1}\right)>0$ (excluded above) or $\lambda<A g^{\prime}\left(U_{1}\right)$. We conclude that $\lambda<A g^{\prime}\left(U_{1}\right)$ is a necessary condition to attain the minimum (A.5) at a positive eigenfunction decaying exponentially at infinity.

We now establish sufficient conditions for the minimum (A.5) to exist.

Lemma A.2 (Conditions for the existence of positive decaying eigenfunctions). Let $F=0, A>0$, and let the nonlinearity $g$ satisfy the hypotheses in Theorem A.1. Let $u_{n}$ be a stationary increasing solution of (1.1) such that $u_{-\infty}=U_{1}(0)$ and $u_{\infty}=U_{3}(0)$. Given an exponentially decaying sequence $w=w_{n}$, we define

$$
J(w)=\frac{\sum_{n}\left[\left(w_{n+1}-w_{n}\right)^{2}+A g^{\prime}\left(u_{n}\right) w_{n}^{2}\right]}{\sum_{n} w_{n}^{2}} .
$$

Let us suppose that there is a sequence $w_{n}$ such that $J\left(w_{n}\right)<A g^{\prime}\left(U_{1}\right)$. Then the infimum

$$
\lambda=\operatorname{Inf}_{\sum_{n} r_{0}^{-2|n|} v_{n}^{2}<\infty} \frac{\sum_{n}\left[\left(v_{n+1}-v_{n}\right)^{2}+A g^{\prime}\left(u_{n}\right) v_{n}^{2}\right]}{\sum_{n} v_{n}^{2}}
$$

is attained at a positive function $V_{n}$ which decays as $r(A, \lambda)^{|n|}$ at infinity, with $0<$ $r(A, \lambda)=\left[2+A g^{\prime}\left(U_{1}\right)-\lambda-\sqrt{\left(-2-A g^{\prime}\left(U_{1}\right)+\lambda\right)^{2}-4}\right] / 2<r_{0}<1$. Now $\lambda=$ $\lambda_{1}(A, 0)$, and $v_{n}$ solves (A.1).

Remark 6 . The value $0<r_{0}<1$ is determined in the proof. Note that $r(A, \lambda)$ is a decreasing function of $A$ but an increasing function of $\lambda$.

Remark 7. We have shown above that $A g^{\prime}\left(U_{2}\right)<\lambda<A g^{\prime}\left(U_{1}\right)$. Thus the smallest eigenvalue shrinks to zero as $A \rightarrow 0$, although we do not have proof that it does so monotonically.

Proof. Clearly $J(w)$ is bounded from below by $A \min _{n}\left(g^{\prime}\left(u_{n}\right)\right)$. We choose $r_{0}=$ $r\left(A, J\left(w_{n}\right)\right) \in(0,1)$ and define $\|w\|_{0}=\sum r_{0}^{-2|n|}\left|w_{n}\right|^{2}$. Let $w^{m}=w_{n}^{m}, m>0$, be a sequence minimizing $J(w):\left\|w^{m}\right\|_{0}<\infty$ and let $J\left(w^{m}\right) \rightarrow \lambda$ when $m \rightarrow \infty$. We replace $w^{m}$ with $v^{m}=v_{n}^{m}=w_{n}^{m} /\left\|w^{m}\right\|_{0}$. Then, $\left\|v^{m}\right\|_{0}=1$ and $J\left(v^{m}\right)=J\left(w^{m}\right) \rightarrow \lambda$. $\left\|v^{m}\right\|_{0}=1$ implies that, uniformly in $m,\left|v_{n}^{m}\right| \leq r_{0}^{|n|}$ and $\sum_{n>n(\epsilon)}\left|v_{n}^{m}\right|^{2}<\epsilon$ for $n(\epsilon)$ large enough. Thus, a subsequence $v^{m}$ tends to some limit $V=V_{n}$ such that $\left|V_{n}\right| \leq r_{0}^{|n|}$ and $\sum_{n}\left|v_{n}^{m}-V_{n}\right|^{2}$ tends to zero as $m$ tends to infinity. Therefore, $J\left(v^{m}\right) \longrightarrow J(V)=\lambda$ and the infimum is attained at the sequence $V=V_{n}$. Moreover, $V=V_{n}$ satisfies the Euler equation (A.1) for the minimization problem, which then implies that $V_{n}$ decays as stated in the lemma. 
On the other hand, $J\left(\left|V_{n}\right|\right) \leq J\left(V_{n}\right)$, and we can choose nonnegative $V_{n}$. But then $\lambda$ has to be the smallest eigenvalue $\lambda_{1}(A, 0)$.

Lemma A.3 (Choice of the sequence $w_{n}$ with $J\left(w_{n}\right)<A g^{\prime}\left(U_{1}\right)$ ). Let $F=0$, $A>0$, and $u_{n}$ be as in Lemma A.2. Let $u(x)$ be the solution of the boundary value problem $d^{2} u / d x^{2}=g(u)$, with $u(-\infty)=U_{1}, u(\infty)=U_{3}$ such that $u(0)=U_{2}$.

- For sufficiently small $A<1$, we have

$$
\left|u_{n+1}-u_{n}\right| \max _{\left[u_{n}, u_{n+1}\right]}\left|g^{\prime \prime}\right|<2 g^{\prime}\left(U_{1}\right) \quad \forall n,
$$

and $w_{n}=u_{n+1}-u_{n}$ satisfies $J\left(w_{n}\right)<A g^{\prime}\left(U_{1}\right)$. An estimation of the appropriate values of $A$ indicates that they should be smaller than

$$
A<\left(\frac{2 g^{\prime}\left(U_{1}\right)}{\max _{\left[U_{1}, U_{3}\right]}\left|g^{\prime \prime}\right| \max _{\mathbb{R}}|d u / d x|}\right)^{2} .
$$

- For $A>2 g^{\prime \prime \prime}\left(U_{1}\right)$, we can choose $w_{n}=0$ for $|n|>M \geq 0, w_{n}=r^{n}$ for $n \geq 0$, and $w_{n}=r^{n+1}$ for $n<0$.

Proof. The function $w_{n}=u_{n+1}-u_{n}$ is a solution of

$$
w_{n+1}-2 w_{n}+w_{n-1}=A \frac{g\left(u_{n+1}\right)-g\left(u_{n}\right)}{u_{n+1}-u_{n}} w_{n}
$$

that decays at infinity as $r(A, 0)^{|n|}$. Multiplying this equation by $w_{n}$ and adding over $n$, we obtain

$$
\sum_{n}\left(\left(w_{n+1}-w_{n}\right)^{2}+A \frac{g\left(u_{n+1}\right)-g\left(u_{n}\right)}{u_{n+1}-u_{n}} w_{n}^{2}\right)=0
$$

This result can be used to calculate $J\left(w_{n}\right)$ :

$$
J\left(w_{n}\right)=A \frac{\sum\left(g^{\prime}\left(u_{n}\right)-\frac{g\left(u_{n+1}\right)-g\left(u_{n}\right)}{u_{n+1}-u_{n}}\right) w_{n}^{2}}{\sum w_{n}^{2}}=-\frac{A}{2} \frac{\sum g^{\prime \prime}\left(\xi_{n}\right) w_{n}^{3}}{\sum w_{n}^{2}},
$$

by the mean value theorem. Thus, $J\left(w_{n}\right) \leq(A / 2) \max _{n}\left(\left|u_{n+1}-u_{n}\right| \max _{\left[u_{n}, u_{n+1}\right]}\left|g^{\prime \prime}\right|\right)$. For sufficiently small $A,\left|u_{n+1}-u_{n}\right| \leq C \sqrt{A}$, so that $J\left(w_{n}\right)<C A^{\frac{3}{2}} \max \left|g^{\prime \prime}\right| / 2<$ $A g^{\prime}\left(U_{1}\right)$. More precisely, for small $A, w_{n}=u_{n+1}-u_{n} \simeq u((n+1) \sqrt{A})-u(n \sqrt{A}) \simeq$ $\sqrt{A} u^{\prime}(\xi)$. Then $\left|w_{n}\right| \leq \max |d u / d x| \sqrt{A}$.

To prove the other case, we observe that $J\left(w_{n}\right)=(r-1)^{2}+A \sum_{i=0}^{\infty} g^{\prime}\left(u_{n}\right) r^{2 n}$ is smaller than $A g^{\prime}\left(u_{1}\right)$, provided that $(1-r) /(1+r)<A g^{\prime \prime \prime}\left(U_{1}\right) /\left[2\left(1-(r(A, 0) r)^{2}\right)\right]$, with $r(A, \lambda)$ defined as in Lemma A.2. The last inequality holds if $A>2 g^{\prime \prime \prime}\left(U_{1}\right)$.

Remark 8. For the FK nonlinearity, the first condition of the lemma holds for $A<0.9$, and the second condition for $A>2$. For intermediate values, numerical simulations show that $w_{n}=u_{n+1}-u_{n}$ satisfies $J\left(w_{n}\right)<A g^{\prime}\left(U_{1}\right)$.

Proposition A.4 (Finite-dimensional approximations). Let $u_{n}(A, F)$ be a stationary solution of (1.1) under the hypotheses in Theorem A.1 for $|F| \leq F_{c}(A)$. Let $\lambda_{1}(A, F)$ be the smallest eigenvalue of the operator $\mathcal{L}(F)$ (linearized about $u_{n}(A, F)$ ) and $\lambda(A, F, N)$ be the smallest eigenvalues of the matrices (3.10). Then, $\lambda(A, F, N) \rightarrow$ $\lambda_{1}(A, F)$ as $N \rightarrow \infty$. As a consequence, if $V>0$ is an eigenfunction associated to $\lambda_{1}(A, F)$ with $\sum_{n} V_{n}^{2}=1$ and if $V(N)>0$ are eigenvectors associated to $\lambda(A, F, N)$ such that $\sum_{n} V_{n}(N)^{2}=1$, then $V(N) \rightarrow V$ as $N \rightarrow \infty$. 
Proof. It follows from the Rayleigh characterizations for the smallest eigenvalues:

$$
\begin{aligned}
& \lambda_{1}(A, F)=\min _{\sum r^{-2|n|} w_{n}^{2}} \frac{\sum_{-\infty}^{\infty}\left[\left(w_{n+1}-w_{n}\right)^{2}+A g^{\prime}\left(u_{n}(A, F)\right) w_{n}^{2}\right]}{\sum_{-\infty}^{\infty} w_{n}^{2}}, \\
& \lambda(A, F, N)=\min \frac{\sum_{-L}^{M}\left[\left(v_{n+1}-v_{n}\right)^{2}+A g^{\prime}\left(u_{n}(A, F)\right) v_{n}^{2}\right]}{\sum_{-L}^{M} v_{n}^{2}} .
\end{aligned}
$$

Letting $w_{n}=v_{n}$ for $n=-L, \ldots, M$ and $w_{n}=0$ otherwise, we see that $\lambda_{1}(A, F) \leq$ $\lambda(A, F, N), N=L+M+1$. Now let $w_{n}$ be an eigenfunction for $\lambda_{1}(A, F)$ such that $\sum_{-\infty}^{\infty} w_{n}^{2}=1$. Then,

$$
\lambda(A, F, N) \leq \frac{\lambda_{1}(A, F)-\sum_{n<-L, n>M}\left[\left(w_{n+1}-w_{n}\right)^{2}+A g^{\prime}\left(u_{n}(A, F)\right) w_{n}^{2}\right]}{\sum_{-L}^{M} w_{n}^{2}} .
$$

We conclude that $\lambda(A, F, N) \rightarrow \lambda_{1}(A, F)$ as $N \rightarrow \infty$. This and the exponential decay of $V$ prove the convergence of the eigenvectors.

A.2. Proof of Theorem A.1. The theorem will be proved in two steps and, for simplicity, in the particular case of periodic $g$. In this case, $U_{3}(F / A)-U_{3}(0)=$ $U_{1}(F / A)-U_{1}(0)$, which allows us to use symmetric sub- and supersolutions. Small modifications are required in the general case.

Step 1: $F_{c}(A)>0$. We use the existence of a positive eigenfunction $v_{n}$ associated with a positive eigenvalue $\lambda_{1}(A, 0)$ to construct stationary supersolutions for (1.1) when $F>0$ is small. The known solution $u_{n}$ provides a stationary subsolution.

We look for a supersolution of the form

$$
w_{n}=u_{n}+(1+\delta)\left(U_{1}\left(\frac{F}{A}\right)-U_{1}(0)\right)+\epsilon v_{n},
$$

with $\delta>0$ to be chosen and $\epsilon, F$ small to be determined. Let us check that

$$
w_{n+1}-2 w_{n}+w_{n-1} \leq g\left(w_{n}\right)-F, \quad w_{\infty}>U_{3}(F / A), w_{-\infty}>U_{1}(F / A)
$$

holds. The conditions at infinity are satisfied for any $\delta>0$. Provided

$$
\left|(1+\delta)\left(U_{1}\left(\frac{F}{A}\right)-U_{1}(0)\right)\right| \leq k \epsilon,
$$

inequality (A.14) holds if

$\epsilon\left(v_{n+1}-2 v_{n}+v_{n-1}\right)<A g^{\prime}\left(u_{n}\right)\left[\epsilon v_{n}+(1+\delta)\left(U_{1}\left(\frac{F}{A}\right)-U_{1}(0)\right)\right]-F+O\left(A \epsilon^{2}\right)$.

Using (A.1), we are left with

$$
F<\epsilon \lambda_{1}(A, 0) v_{n}+A(1+\delta) g^{\prime}\left(u_{n}\right)\left(U_{1}\left(\frac{F}{A}\right)-U_{1}(0)\right) .
$$

Now, $U_{1}(F / A)=g^{-1}(F / A)$, the inverse being taken near $U_{1}(0)$, in the region with $g^{\prime}>0$. Using $g^{-1}(x) \sim g^{-1}\left(x_{0}\right)+\left(g^{-1}\right)^{\prime}\left(x_{0}\right)\left(x-x_{0}\right)$, we obtain

$$
U_{1}\left(\frac{F}{A}\right)=g^{-1}\left(\frac{F}{A}\right) \sim U_{1}(A, 0)+\frac{F}{A g^{\prime}\left(U_{1}(0)\right)} .
$$


Thus, the condition for $w_{n}$ to be a supersolution is

$$
F<\epsilon \lambda_{1}(A, 0) v_{n}+g^{\prime}\left(u_{n}\right) \frac{1+\delta}{g^{\prime}\left(U_{1}(0)\right)} F .
$$

Let $M$ be sufficiently large. We distinguish two different ranges of indices $n$ :

- For $|n|>M, g^{\prime}\left(u_{n}\right)>0$ and $v_{n} \ll 1$. Then the right-hand side of the previous inequality is dominated by the second term. We choose $\delta$ large enough to ensure $F<g^{\prime}\left(u_{n}\right)(1+\delta) F / g^{\prime}\left(U_{1}(0)\right)$, that is, $1+\delta>g^{\prime}\left(U_{1}(0)\right) / g^{\prime}\left(u_{M}\right)$.

- For small $|n|, g^{\prime}\left(u_{n}\right)<0$. The previous inequality is satisfied, provided we choose $F$ so small that

$$
\left(1+\left|g^{\prime}\left(u_{n}\right)\right| \frac{1+\delta}{g^{\prime}\left(U_{1}(0)\right)}\right) F<\epsilon \lambda_{1}(A, 0) v_{n}
$$

for a fixed value of $\delta$.

With these choices, $w_{n}$ satisfies (A.14). Note that these choices are compatible with condition (A.15). Using (A.16), (A.15) becomes $(1+\delta) F /\left[A g^{\prime}\left(U_{1}(0)\right)\right]<k \epsilon$. This holds for small enough $F$.

Let $F>0$ be small enough for a $w_{n}$ defined in (A.13) to be a supersolution with $\delta, \epsilon$ adequately selected. Now, let $h_{n}(t)$ be a solution to (1.1) for such $F>0$ with initial datum $h_{n}(0)$ satisfying $u_{n}<h_{n}(0)<w_{n}$. Then, $u_{n}<h_{n}(t)<w_{n}$ for all $t>0$. Therefore, propagation is excluded and the solutions are pinned.

Stationary solutions $u_{n}(A, F)$ for such $F>0$ can be obtained as long time limits of solutions $h_{n}(t)$ to $(1.1)$ when $h_{n}(0)$ is increasing, tends exponentially to $U_{1}(A, F)$ (resp., $U_{3}(A, F)$ ) at $-\infty$ (resp., $\left.\infty\right)$, and $u_{n}<h_{n}(0)<w_{n}$. We conclude that $F_{c}(A)>0$.

Step 2: $\lambda_{1}(A, F)>0$ for $|F|<F_{c}(A)$ and $\lambda_{1}\left(A, F_{c}(A)\right)=0$. To fix ideas, we take $F>0$. The case $F<0$ follows by symmetry. From Step 1, we know that $F_{c}(A)>0$, and there are stationary solutions $u_{n}(A, F)$ of (1.1) existing for $F>0$ small that are increasing from $U_{1}(F / A)$ to $U_{3}(F / A)$.

In an analogous way as we $\operatorname{did}$ for $F=0$, we get

$$
\lambda_{1}(A, F)=\min _{\sum r_{0}^{-2|n|} w_{n}^{2}<\infty} \frac{\sum_{n}\left[\left(w_{n+1}-w_{n}\right)^{2}+A g^{\prime}\left(u_{n}(A, F)\right) w_{n}^{2}\right]}{\sum w_{n}^{2}} .
$$

This formula defines $\lambda_{1}(A, F)$ as a continuous function of $F$. That $\lambda_{1}(A, 0)>0$ implies $\lambda_{1}(A, F)>0$ up to some $F_{c}$ at which $\lambda_{1}\left(A, F_{c}\right)=0$. As long as $\lambda_{1}\left(A, F_{1}\right)>0$, we can obtain stationary solutions for $F>F_{1}$ (close to $F_{1}$ ), as done in Step 1. This procedure cannot continue forever since such stationary solutions do not exist for $F$ close to $A$ : eventually $g(U)=F / A$ ceases to have three solutions, and the stationary wave fronts cannot be constructed. Thus, we must reach a value $F_{c}$ at which $\lambda_{1}\left(A, F_{c}\right)=0$.

Acknowledgments. The authors are indebted to J. M. Vega for a critical reading of the manuscript and helpful comments, and to V. Hakim for pointing out to them the relevance of [7]. A. C. thanks S. P. Hastings and J. B. McLeod for fruitful discussions.

\section{REFERENCES}

[1] A. Amann, A. Wacker, L. L. Bonilla, And E. Schöll, Dynamic scenarios of multistable switching in semiconductor superlattices, Phys. Rev. E, 63 (2001), paper 066207. 
[2] L. L. Bonilla, Stable probability densities and phase transitions for mean-field models in the thermodynamic limit, J. Statist. Phys., 46 (1987), pp. 659-678.

[3] L. L. Bonilla, J. Galán, J. A. Cuesta, F. C. Martínez, And J. M. Molera, Dynamics of electric field domains and oscillations of the photocurrent in a simple superlattice model, Phys. Rev. B, 50 (1994), pp. 8644-8657.

[4] O. M. Braun and Yu. S. Kivshar, Nonlinear dynamics of the Frenkel-Kontorova model, Phys. Rep., 306 (1998), pp. 1-108.

[5] P. C. BRessloff AND G. Rowlands, Exact travelling wave solutions of an "integrable" discrete reaction-diffusion equation, Phys. D, 106 (1997), pp. 255-269.

[6] A. E. Bugrim, A. M. Zhabotinsky, And I. R. Epstein, Calcium waves in a model with a random spatially discrete distribution of $\mathrm{Ca}^{2+}$ release sites, Biophys. J., 73 (1997), pp. 2897-2906.

[7] J. W. CAHN, Theory of crystal growth and interface motion in crystalline materials, Acta Metallurgica, 8 (1960), pp. 554-562.

[8] A. Carpio, S. J. Chapman, S. Hastings, and J. B. McLeod, Wave solutions for a discrete reaction-diffusion equation, European J. Appl. Math., 11 (2000), pp. 399-412.

[9] A. Carpio, L. L. Bonilla, A. Wacker, and E. Schöll, Wave fronts may move upstream in doped semiconductor superlattices, Phys. Rev. E, 61 (2000), pp. 4866-4876.

[10] A. Carpio and L. L. Bonilla, Wave front depinning transition in discrete one-dimensional reaction-diffusion systems, Phys. Rev. Lett., 86 (2001), pp. 6034-6037.

[11] A. Carpio, L. L. Bonilla, and G. Dell'Acqua, Motion of wave fronts in semiconductor superlattices, Phys. Rev. E, 64 (2001), paper 036204.

[12] A. Carpio, L. L. Bonilla, And A. Luzón, Effects of disorder on the wave front depinning transition in spatially discrete systems, Phys. Rev. E, 65 (2002), paper 035207(R)

[13] P. M. Chaikin and T. C. Lubensky, Principles of Condensed Matter Physics, Cambridge University Press, Cambridge, UK, 1995.

[14] T. Erneux and G. Nicolis, Propagating waves in discrete reaction-diffusion systems, Phys. D, 67 (1993), pp. 237-244.

[15] G. FÁth, Propagation failure of traveling waves in a discrete bistable medium, Phys. D, 116 (1998), pp. 176-190.

[16] S. Flach, Y. Zolotaryuk, and K. Kladko, Moving lattice kinks and pulses: An inverse method, Phys. Rev. E, 59 (1999), pp. 6105-6115.

[17] J. Frenkel and T. Kontorova, On the theory of plastic deformation and twinning, J. Phys. USSR, 13 (1938), pp. 1-10.

[18] E. Gerde And M. Marder, Friction and fracture, Nature, 413 (2001), pp. 285-288.

[19] G. Grüner, The dynamics of charge-density waves, Rev. Modern Phys., 60 (1988), pp. 11291181.

[20] V. HAKIM AND K. MALLICK, Exponentially small splitting of separatrices, matching in the complex plane and Borel summation, Nonlinearity, 6 (1993), pp. 57-70.

[21] R. Hobart, Peierls-barrier minima, J. Appl. Phys., 36 (1965), pp. 1948-1952.

$[22]$ V. L. Indenbom, Mobility of dislocations in the Frenkel-Kontorova model, Soviet Phys.Crystallogr., 3 (1959), pp. 193-201 (translated from Kristallografiya, 3 (1958), pp. 197206).

[23] J. P. KeEner, Propagation and its failure in coupled systems of discrete excitable cells, SIAM J. Appl. Math., 47 (1987), pp. 556-572.

[24] J. P. Keener and J. Sneyd, Mathematical Physiology, Springer, New York, 1998.

[25] P. G. Kevrekidis, C. K. R. T. Jones, and T. Kapitula, Exponentially small splitting of heteroclinic orbits: From the rapidly forced pendulum to discrete solitons, Phys. Lett. A, 269 (2000), pp. 120-129.

[26] P. G. Kevrekidis, I. G. Kevrekidis, And A. R. Bishop, Propagation failure, universal scalings and Goldstone modes, Phys. Lett. A, 279 (2001), pp. 361-369.

[27] J. R. King And S. J. Chapman, Asymptotics beyond all orders and Stokes lines in nonlinear differential-difference equations, European J. Appl. Math., 12 (2001), pp. 433-463.

[28] K. Kladko, I. Mitkov, And A. R. Bishop, Universal scaling of wave propagation failure in arrays of coupled nonlinear cells, Phys. Rev. Lett., 84 (2000), pp. 4505-4508.

[29] J. Miles, On Faraday resonance of a viscous liquid, J. Fluid Mech., 395 (1999), pp. 321-325.

[30] I. Mitkov, K. Kladko, and J. E. Pearson, Tunable pinning of bursting waves in extended systems with discrete sources, Phys. Rev. Lett., 81 (1998), pp. 5453-5456.

[31] F. R. N. Nabarro, Dislocations in a simple cubic lattice, Proc. Phys. Soc. London, 59 (1947), pp. 256-272.

[32] F. R. N. NABarro, Theory of Crystal Dislocations, Oxford University Press, Oxford, UK, 1967. 
[33] R. Peierls, The size of a dislocation, Proc. Phys. Soc. London, 52 (1940), pp. 34-37.

[34] V. H. Schmidt, Exact solution in the discrete case for solitons propagating in a chain of harmonically coupled particles lying in double-minimum potential wells, Phys. Rev. B, 20 (1979), pp. 4397-4405.

[35] L. I. Slepyan, Dynamics of a crack in a lattice, Sov. Phys. Dokl., 26 (1981), pp. 538-540 (translated from Dokl. Akad. Nauk SSSR, 258 (1981), pp. 561-564).

[36] J. M. Speight And R. S. WARD, Kink dynamics in a novel discrete sine-Gordon system, Nonlinearity, 7 (1994), pp. 475-484.

[37] J. M. Speight, A discrete $\phi^{4}$ system without a Peierls-Nabarro barrier, Nonlinearity, 10 (1997), pp. $1615-1625$.

[38] J. M. Speight, Topological discrete kinks, Nonlinearity, 12 (1999), pp. 1373-1387.

[39] H. S. J. van der Zant, T. P. Orlando, S. Watanabe, and S. H. Strogatz, Kink propagation in a discrete system: Observation of phase locking to linear waves, Phys. Rev. Lett., 74 (1995), pp. 174-177.

[40] B. ZINNER, Existence of traveling wave front solutions for the discrete Nagumo equation, J. Differential Equations, 96 (1992), pp. 1-27. 